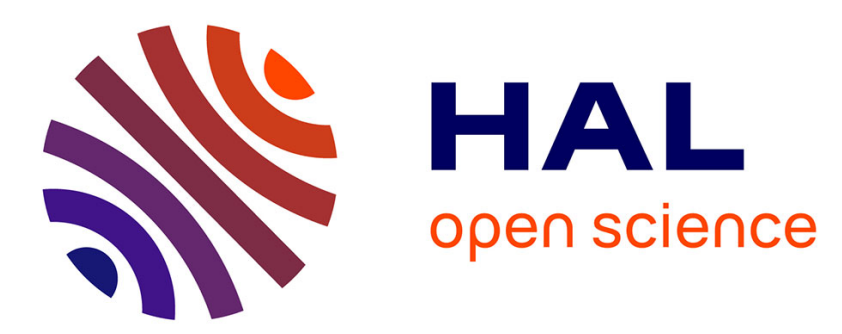

\title{
A multi well-balanced scheme for the shallow water MHD system with topography
}

François Bouchut, Xavier Lhébrard

\section{To cite this version:}

François Bouchut, Xavier Lhébrard. A multi well-balanced scheme for the shallow water MHD system with topography. Numerische Mathematik, 2017, 136 (4), pp.875-905. 10.1007/s00211-017-0865-y . hal-01131297v3

\section{HAL Id: hal-01131297 \\ https://hal.science/hal-01131297v3}

Submitted on 1 Dec 2016

HAL is a multi-disciplinary open access archive for the deposit and dissemination of scientific research documents, whether they are published or not. The documents may come from teaching and research institutions in France or abroad, or from public or private research centers.
L'archive ouverte pluridisciplinaire HAL, est destinée au dépôt et à la diffusion de documents scientifiques de niveau recherche, publiés ou non, émanant des établissements d'enseignement et de recherche français ou étrangers, des laboratoires publics ou privés. 


\title{
A multi well-balanced scheme for the shallow water MHD system with topography
}

\author{
François Bouchut*, Xavier Lhébrard*
}

\begin{abstract}
The shallow water magnetohydrodynamic system involves several families of physically relevant steady states. In this paper we design a wellbalanced numerical scheme for the one-dimensional shallow water magnetohydrodynamic system with topography, that resolves exactly a large range of steady states. Two variants are proposed with slightly different families of preserved steady states. They are obtained by a generalized hydrostatic reconstruction algorithm involving the magnetic field and with a cutoff parameter to remove singularities. The solver is positive in height and semi-discrete entropy satisfying, which ensures the robustness of the method.
\end{abstract}

Keywords: Shallow water magnetohydrodynamics, topography, well-balanced scheme, hydrostatic reconstruction, semi-discrete entropy inequality.

Mathematics Subject Classification: 76W05, 76M12, 35L65

\section{Introduction}

The shallow water magnetohydrodynamic (SWMHD) system has been introduced in [25] to describe the thin layer evolution of the solar tachocline. It is written in $2 \mathrm{~d}$ in the tangent plane approximation as

$$
\begin{gathered}
\partial_{t} h+\nabla \cdot(h \mathbf{u})=0, \\
\partial_{t}(h \mathbf{u})+\nabla \cdot(h \mathbf{u} \otimes \mathbf{u}-h \mathbf{b} \otimes \mathbf{b})+\nabla\left(g h^{2} / 2\right)+g h \nabla z+f h \mathbf{u}^{\perp}=0, \\
\partial_{t}(h \mathbf{b})+\nabla \cdot(h \mathbf{b} \otimes \mathbf{u}-h \mathbf{u} \otimes \mathbf{b})+\mathbf{u} \nabla \cdot(h \mathbf{b})=0,
\end{gathered}
$$

where $g>0$ is the gravity constant, $h \geq 0$ is the thickness of the fluid, $\mathbf{u}=(u, v)$ is the velocity, $\mathbf{b}=(a, b)$ is the magnetic field, $z(x)$ is the topography, $f(x)$ is the Coriolis parameter, and $\mathbf{u}^{\perp}$ denotes the vector obtained from $\mathbf{u}$ by a rotation of angle $\pi / 2$. The notation $\nabla \cdot(\mathbf{b} \otimes \mathbf{u})$ is for the vector with index $i$ given by $\sum_{j} \partial_{j}\left(b_{i} u_{j}\right)$. The system has to be completed with the entropy (energy) inequality

$$
\begin{aligned}
& \partial_{t}\left(\frac{1}{2} h|\mathbf{u}|^{2}+\frac{1}{2} g h^{2}+\frac{1}{2} h|\mathbf{b}|^{2}+g h z\right) \\
& +\nabla \cdot\left(\left(\frac{1}{2} h|\mathbf{u}|^{2}+g h^{2}+\frac{1}{2} h|\mathbf{b}|^{2}+g h z\right) \mathbf{u}-h \mathbf{b}(\mathbf{b} \cdot \mathbf{u})\right) \leq 0,
\end{aligned}
$$

*Université Paris-Est, Laboratoire d'Analyse et de Mathématiques Appliquées (UMR 8050), CNRS, UPEM, UPEC, F-77454, Marne-la-Vallée, France (Francois.Bouchut@upem.fr), (Xavier.Lhebrard@u-pem.fr) 
that becomes an equality in the absence of shocks. We recall that the extra term $\mathbf{u} \nabla \cdot(h \mathbf{b})$ in the induction equation (1.3), that has been proposed in [19], is put for $2 \mathrm{~d}$ numerical purposes only, while the physically relevant situation is to have the magnetic divergence constraint $\nabla \cdot(h \mathbf{b})=0$. Adding this term enables to deal with the divergence constraint numerically. The method has been proved to be robust in the MHD case, see [23,31]. Other methods to deal with the divergence constraint are for example [5, 18].

Multidimensional simulations of the SWMHD system have been performed in $[28,29,30]$. As for the compressible MHD system, one-dimensional solvers that are accurate on contact waves are needed in order to reduce significantly the numerical diffusion in complex and multidimensional settings, that generically involve Alfven waves, see for example [23, 4]. At the same time, the robustness of the scheme must be maintained. This is why robust and accurate schemes in the one-dimensional situation are needeed, and it is the subject of this paper.

In the one and a half dimensional setting, i.e. if dependency is only in one spatial variable $x$, the system (1.1)-(1.3) simplifies to

$$
\begin{aligned}
\partial_{t} h+\partial_{x}(h u) & =0, \\
\partial_{t}(h u)+\partial_{x}\left(h u^{2}+P\right)+g h \partial_{x} z-f h v & =0, \\
\partial_{t}(h v)+\partial_{x}\left(h u v+P_{\perp}\right)+f h u & =0, \\
\partial_{t}(h a)+u \partial_{x}(h a) & =0, \\
\partial_{t}(h b)+\partial_{x}(h b u-h a v)+v \partial_{x}(h a) & =0,
\end{aligned}
$$

with

$$
P=g \frac{h^{2}}{2}-h a^{2}, \quad P_{\perp}=-h a b,
$$

and the energy inequality (1.4) becomes

$$
\begin{aligned}
& \partial_{t}\left(\frac{1}{2} h\left(u^{2}+v^{2}\right)+\frac{1}{2} g h^{2}+\frac{1}{2} h\left(a^{2}+b^{2}\right)+g h z\right) \\
& +\partial_{x}\left(\left(\frac{1}{2} h\left(u^{2}+v^{2}\right)+g h^{2}+\frac{1}{2} h\left(a^{2}+b^{2}\right)+g h z\right) u-h a(a u+b v)\right) \leq 0 .
\end{aligned}
$$

According to [20], the eigenvalues of the system (1.5)-(1.9) are $u, u \pm|a|, u \pm$ $\sqrt{a^{2}+g h}$. The associated waves are called respectively material (or divergence) waves, Alfven waves and magnetogravity waves. It is classical in shallow water systems to consider the topography $z$ as an additional variable to the system, satisfying $\partial_{t} z=0$. In this setting there is an additional eigenvalue which is 0 , and we shall call the associated wave the topography wave. The presence of the zero-order Coriolis terms proportional to $f$ induces indeed more complex nonlinear waves [34]. These are studied numerically in [35]. In the present work, from now on we shall always assume that $f \equiv 0$.

The system (1.5)-(1.9) is nonconservative in the variables $h a, h b$. However, $h a$ jumps only through the material contacts, where $u$ and $v$ are continuous. Therefore, there is indeed no ambiguity in the non conservative products $u \partial_{x}(h a)$ and $v \partial_{x}(h a)$, that are well-defined. Concerning the nonconservative term $h \partial_{x} z$ in (1.6), it is well-defined for continuous topography $z$. Piecewise constant discontinuous $z$ is considered however for discrete approximations. 
A striking property of the system (1.5)-(1.9) is that four out of six of the waves are contact discontinuities, corresponding to linearly degenerate eigenvalues: the material contacts associated to the eigenvalue $u$, the left Alfven contacts associated to $u-|a|$, the right Alfven contacts associated to $u+|a|$, and the topography contacts associated to the eigenvalue 0 . Resonance can occur, which means that these waves can collapse. It happens in particular when $u=0$ or $u \pm|a|=0$.

Well-balanced finite volume schemes for solving shallow water type models with topography have been extensively developed, see [8] and the references therein. A main principle in such schemes is to resolve exactly some steady states, in order to reduce significantly the numerical error. The same question arises for hydrodynamic systems without topography, when linearly degenerate eigenvalues are involved. Indeed, in the numerical simulation of conservation laws, shocks are generally better resolved than contact discontinuities because of their compressive nature. This is why it is important to resolve well the contact discontinuities, that do not benefit of any compressive effect. In the SWMHD system (1.5)-(1.9), we have at the same time "dynamic" linearly degenerate eigenvalues (material and Alfven contact waves), and the "static" linearly degenerate eigenvalue (steady topography contact waves). The aim of this paper is to build a well-balanced scheme for the SWMHD system (1.5)-(1.9) that is accurate on all these contact waves. Two variants are proposed. Our work follows [10], where we built an entropy satisfying approximate Riemann solver for the SWMHD system without topography that is accurate on all contact waves.

A generic tool for building well-balanced schemes that we use is the hydrostatic reconstruction method, that has been introduced in [1]. One of its strengths is that it enforces a semi-discrete entropy inequality, ensuring the robustness of the scheme and the computation of entropic shocks. Several variants and extensions have been proposed in $[8,14,11,12,9]$, and a fully discrete entropy inequality is established in [2]. Other approaches are the Roe method $[3,27,26,15,13]$, the approximate Riemann solver method [24, 16, 7, 22]. A system similar to ours with several families of steady states is treated in particular in [21]. Central schemes are used also, and can handle multi steady states [17]. Higher-order extensions are reviewed in [32].

The paper is organized as follows. In Section 2 we describe the steady states of the SWMHD system with topography. In Section 3 we write down our two numerical schemes, with numerical fluxes that involve very particular reconstruction procedures, and our main results Theorems 3.1 and 3.2. Section 4 is devoted to the proofs of these theorems. In Section 5 we perform numerical tests, and finally Section 6 states our conclusions.

\section{Steady states}

As mentioned above, the system with topography (1.5)-(1.9) with $f \equiv 0$ has four linearly degenerate eigenvalues $u-|a|, u, u+|a|$ and 0 , that can be resonant. We would like to build a scheme that is well-balanced for some contact waves for the eigenvalue 0 , that are in particular steady states. Several cases can be considered. For each of them, it is straightforward to check that the following relations define steady states. 
- Non-resonant case $(u \neq 0$ and $u \pm a \neq 0)$. The relations are

$$
\begin{gathered}
h u=c s t(\neq 0), \quad h a=c s t(\neq \pm h u), \quad v=c s t, \quad b=c s t, \\
\frac{u^{2}}{2}-\frac{a^{2}}{2}+g(h+z)=c s t .
\end{gathered}
$$

As is usual in the classical shallow water system, we shall not consider these steady states for the well-balanced property, because they are too complicate to handle. When possible, solving these steady states generally improves the accuracy, see $[11,33,6]$.

- Material resonant case $(u=0$ and $a \neq 0)$. The differential relations are

$$
\begin{gathered}
u=0, \quad v=c s t, \quad h a b=c s t, \\
\partial_{x}\left(g \frac{h^{2}}{2}-h a^{2}\right)+g h \partial_{x} z=0 .
\end{gathered}
$$

Note that in contrast with the other cases, the second line in (2.2) is not integrable. It implies that for discontinuous data, this differential relation can have different possible interpretations in terms of nonconservative products. The situation is the same in [21].

We shall thus consider two particular subfamilies of steady states from (2.2). The first is characterized by the relation $\sqrt{h} a=c s t$, which yields

$$
u=0, \quad v=c s t, \quad h+z=c s t, \quad \sqrt{h} a=c s t(\neq 0), \quad \sqrt{h} b=c s t .
$$

The second subfamily of steady states from (2.2) is characterized by the relation $h a=c s t$, that leads to the steady states

$$
u=0, \quad v=c s t, \quad h a=c s t(\neq 0), \quad b=c s t, \quad h-\frac{a^{2}}{2 g}+z=c s t .
$$

These are indeed the limit of (2.1) when $h u \rightarrow 0$.

- Alfven resonant case $(u \neq 0$ and $u \pm a=0)$. The relations are

$$
h u=c s t(\neq 0), \quad h a=\mp h u, \quad h+z=c s t, \quad v \pm b=c s t .
$$

- Material and Alfven resonant case $(u=a=0)$. The relations are

$$
u=0, \quad a=0, \quad h+z=c s t .
$$

\section{Hydrostatic reconstruction scheme and main results}

In this section we define our two variants of hydrostatic reconstruction scheme for the SWMHD system (1.5)-(1.9), and state their properties.

A finite volume scheme for the nonconservative system (1.5)-(1.9) with $f \equiv 0$ can be written

$$
U_{i}^{n+1}=U_{i}^{n}-\frac{\Delta t}{\Delta x_{i}}\left(F_{l}\left(U_{i}^{n}, U_{i+1}^{n}, \Delta z_{i+1 / 2}\right)-F_{r}\left(U_{i-1}^{n}, U_{i}^{n}, \Delta z_{i-1 / 2}\right)\right),
$$


where

$$
U=(h, h u, h v, h a, h b),
$$

and as usual $n$ stands for the time index, $i$ for the space location, and $\Delta z_{i+1 / 2}=$ $z_{i+1}-z_{i}$. Thus we need to define the left and right numerical fluxes $F_{l}\left(U_{l}, U_{r}, \Delta z\right)$, $F_{r}\left(U_{l}, U_{r}, \Delta z\right)$, for all left and right values $U_{l}, U_{r}, z_{l}, z_{r}$ with $\Delta z=z_{r}-z_{l}$. They are constructed via the hydrostatic reconstruction method of [1].

\subsection{First scheme, associated to the steady states (2.3)}

Our first scheme resolves the steady states (2.3). Denoting the left and right states by $U_{l}=\left(h_{l}, h_{l} u_{l}, h_{l} v_{l}, h_{l} a_{l}, h_{l} b_{l}\right), U_{r}=\left(h_{r}, h_{r} u_{r}, h_{r} v_{r}, h_{r} a_{r}, h_{r} b_{r}\right)$, we define the reconstructed heights

$$
h_{l}^{\#}=\left(h_{l}-(\Delta z)_{+}\right)_{+}, \quad h_{r}^{\#}=\left(h_{r}-(-\Delta z)_{+}\right)_{+},
$$

with the notation $x_{+} \equiv \max (0, x)$. We also define new reconstructed magnetic states

$$
\begin{array}{ll}
a_{l}^{\#}=\kappa_{l} a_{l}, & a_{r}^{\#}=\kappa_{r} a_{r}, \\
b_{l}^{\#}=\kappa_{l} b_{l}, & b_{r}^{\#}=\kappa_{r} b_{r},
\end{array}
$$

with

$$
\kappa_{l}=\min \left(\sqrt{\frac{h_{l}}{h_{l}^{\#}}}, \gamma\right), \quad \kappa_{r}=\min \left(\sqrt{\frac{h_{r}}{h_{r}^{\#}}}, \gamma\right),
$$

and where $\gamma \geq 1$ is a cutoff parameter used to prevent from getting infinite values in (3.6) when $h_{l / r}^{\#}$ vanish. In the special case $h_{l}=0$ (respectively $h_{r}=0$ ), we have $h_{l}^{\#}=0$ (respectively $h_{r}^{\#}=0$ ) and we set by convention $\kappa_{l}=1$ (respectively $\kappa_{r}=1$ ). We define then the left and right reconstructed states as

$U_{l}^{\#}=\left(h_{l}^{\#}, h_{l}^{\#} u_{l}, h_{l}^{\#} v_{l}, h_{l}^{\#} a_{l}^{\#}, h_{l}^{\#} b_{l}^{\#}\right), \quad U_{r}^{\#}=\left(h_{r}^{\#}, h_{r}^{\#} u_{r}, h_{r}^{\#} v_{r}, h_{r}^{\#} a_{r}^{\#}, h_{r}^{\#} b_{r}^{\#}\right)$.

Note that we use the notation \# instead of * in order to avoid confusions with intermediate states of Riemann solvers. Then the numerical fluxes are defined by

$$
\begin{aligned}
& F_{l}\left(U_{l}, U_{r}, \Delta z\right)=\mathcal{F}_{l}\left(U_{l}^{\#}, U_{r}^{\#}\right) \\
& +\left(0, g \frac{h_{l}^{2}}{2}-g \frac{h_{l}^{\# 2}}{2}, 0,\left(\kappa_{l}(h a)_{l}^{\#}-(h a)_{l}\right) u_{l},\left(\kappa_{l}(h a)_{l}^{\#}-(h a)_{l}\right) v_{l}\right) \\
& +\left(\kappa_{l}-1\right)\left(0,0,0, \mathcal{F}_{l}^{h a}\left(U_{l}^{\#}, U_{r}^{\#}\right), \mathcal{F}_{l}^{h b}\left(U_{l}^{\#}, U_{r}^{\#}\right)\right) \\
& +\mathcal{F}^{h}\left(U_{l}^{\#}, U_{r}^{\#}\right)\left(0,0,0, \frac{a_{l}}{2}\left(1-\kappa_{l}^{2}\right), \frac{b_{l}}{2}\left(1-\kappa_{l}^{2}\right)\right), \\
& F_{r}\left(U_{l}, U_{r}, \Delta z\right)=\mathcal{F}_{r}\left(U_{l}^{\#}, U_{r}^{\#}\right) \\
& +\left(0, g \frac{h_{r}^{2}}{2}-g \frac{h_{r}^{\# 2}}{2}, 0,\left(\kappa_{r}(h a)_{r}^{\#}-(h a)_{r}\right) u_{r},\left(\kappa_{r}(h a)_{r}^{\#}-(h a)_{r}\right) v_{r}\right) \\
& +\left(\kappa_{r}-1\right)\left(0,0,0, \mathcal{F}_{r}^{h a}\left(U_{l}^{\#}, U_{r}^{\#}\right), \mathcal{F}_{r}^{h b}\left(U_{l}^{\#}, U_{r}^{\#}\right)\right) \\
& +\mathcal{F}^{h}\left(U_{l}^{\#}, U_{r}^{\#}\right)\left(0,0,0, \frac{a_{r}}{2}\left(1-\kappa_{r}^{2}\right), \frac{b_{r}}{2}\left(1-\kappa_{r}^{2}\right)\right),
\end{aligned}
$$


where $\mathcal{F}_{l}$ and $\mathcal{F}_{r}$ are the numerical fluxes of [10] associated to the problem without topography, and $\mathfrak{F}^{h}$ is its common left/right height flux. Note that

$$
\Delta z=0 \text { implies }\left\{\begin{array}{l}
U_{l}^{\#}=U_{l}, U_{r}^{\#}=U_{r}, \\
F_{l}\left(U_{l}, U_{r}, 0\right)=\mathcal{F}_{l}\left(U_{l}, U_{r}\right), F_{r}\left(U_{l}, U_{r}, 0\right)=\mathcal{F}_{r}\left(U_{l}, U_{r}\right),
\end{array}\right.
$$

which means that the numerical fluxes extend the ones of the homogeneous solver.

Theorem 3.1. The scheme (3.1) with the numerical fluxes $F_{l}, F_{r}$ defined by (3.8), (3.9) with the reconstruction (3.3)-(3.7) satisfies the following properties.

(i) It is conservative in the variables $h$ and $h v$,

(ii) It is consistent with (1.5)-(1.9) for smooth solutions,

(iii) It keeps the positivity of $h$ under the CFL condition $\Delta t A\left(U_{l}^{\#}, U_{r}^{\#}\right) \leq$ $\frac{1}{2} \min \left(\Delta x_{l}, \Delta x_{r}\right)$ with $A(.,$.$) the maximum speed of the homogeneous solver,$ defined by [10, eq. (4.8)],

(iv) It satisfies a semi-discrete energy inequality associated to (1.11),

(v) It is well-balanced with respect to steady material and Alfven contact discontinuities without jump in topography,

(vi) It is well-balanced with respect to the steady states (2.6) corresponding to material and Alfven resonance.

(vii) It is well-balanced with respect to the steady states (2.3) that satisfy

$$
\max \left(\sqrt{\frac{h_{l}}{h_{r}}}, \sqrt{\frac{h_{r}}{h_{l}}}\right) \leq \gamma
$$

The proof of Theorem 3.1 is given in Subsection 4.1, and we give here some comments on this result.

- The formulas (3.8), (3.9) for the numerical fluxes are defined exactly so that the proof of the entropy inequality is an identity. Then it follows that the scheme is consistent.

- The particular values (3.6) of $\kappa_{l}, \kappa_{r}$ are involved only in the well-balanced property (vii), and do not matter for the other properties. We only need that their value is 1 when $\Delta z=0$. In particular, if $\gamma=1$ we get $\kappa_{l} \equiv$ $\kappa_{r} \equiv 1$, but then we loose the property (vii) since the condition (3.11) then selects only the trivial constant states. In general one should choose $\gamma$ large enough to include relevant steady states in the condition (3.11), but not too large to avoid large values of $\kappa_{l}, \kappa_{r}$ when $h_{l}^{\#}$ or $h_{r}^{\#}$ is small due to a large topography jump $\Delta z$.

One can use also different formulas like

$$
\kappa_{l}=\min \left(\frac{h_{l}}{h_{l}^{\#}}, \gamma\right), \quad \kappa_{r}=\min \left(\frac{h_{r}}{h_{r}^{\#}}, \gamma\right),
$$


the idea being to have, if $\gamma$ is large enough, $\kappa_{l}=h_{l} / h_{l}^{\#}, \kappa_{r}=h_{r} / h_{r}^{\#}$, $h_{l}^{\#} a_{l}^{\#}=h_{l} a_{l}, h_{r}^{\#} a_{r}^{\#}=h_{r} a_{r}$. However, with (3.12) or with (3.6), the scheme does not preserve the relation $h a=c s t$, because of the form (3.8), (3.9) of the numerical fluxes. This is the reason why we propose another reconstruction in the next subsection.

\subsection{Second scheme, associated to the steady states (2.4)}

Our second scheme resolves the steady states (2.4). It aims at the same time to keep the relation $h a=c s t$ if it is satisfied initially. The reconstructed states are defined as follows for $U_{l}=\left(h_{l}, h_{l} u_{l}, h_{l} v_{l}, h_{l} a_{l}, h_{l} b_{l}\right), U_{r}=\left(h_{r}, h_{r} u_{r}, h_{r} v_{r}, h_{r} a_{r}, h_{r} b_{r}\right)$.

We consider a cutoff parameter $\gamma \geq 1$ and we set $h_{l}^{\#}=0$ if $h_{l}=0$, otherwise for $h_{l}>0$

$$
\left\{\begin{array}{l}
h_{l}^{\#}-\frac{a_{l}^{2}}{2 g} \min \left(\frac{h_{l}}{h_{l}^{\#}, \gamma}\right)^{2}=h_{l}-\frac{a_{l}^{2}}{2 g}+z_{l}-z^{\#} \quad \text { if } h_{l}+\left(\gamma^{2}-1\right) \frac{a_{l}^{2}}{2 g} \geq z^{\#}-z_{l}, \\
h_{l}^{\#}=0 \text { otherwise }
\end{array}\right.
$$

with

$$
z^{\#}=\max \left(z_{l}, z_{r}\right) .
$$

Indeed, the function $h \mapsto h-\left(a_{l}^{2} / 2 g\right) \min \left(h_{l} / h, \gamma\right)^{2}$ is increasing on $[0, \infty)$, and the condition on the data in (3.13) is for having a solution $h_{l}^{\#} \geq 0$ to the equation in the first line. In the case there is no nonnegative solution, we set $h_{l}^{\#}=0$. Similarly we set on the right for $h_{r}>0$

$$
\left\{\begin{array}{l}
h_{r}^{\#}-\frac{a_{r}^{2}}{2 g} \min \left(\frac{h_{r}}{h_{r}^{\#}}, \gamma\right)^{2}=h_{r}-\frac{a_{r}^{2}}{2 g}+z_{r}-z^{\#} \quad \text { if } h_{r}+\left(\gamma^{2}-1\right) \frac{a_{r}^{2}}{2 g} \geq z^{\#}-z_{r}, \\
h_{r}^{\#}=0 \text { otherwise. }
\end{array}\right.
$$

Then we have in any case

$$
0 \leq h_{l}^{\#} \leq h_{l}, \quad 0 \leq h_{r}^{\#} \leq h_{r} .
$$

Isolating the case when $h_{l}^{\#} \geq h_{l} / \gamma$, definition (3.13) is found equivalent to set for $h_{l}>0$

$$
\left\{\begin{array}{l}
h_{l}^{\#}-\frac{(h a)_{l}^{2}}{2 g\left(h_{l}^{\#}\right)^{2}}=h_{l}-\frac{a_{l}^{2}}{2 g}+z_{l}-z^{\#}, \quad \text { if }\left(1-\frac{1}{\gamma}\right) h_{l}+\left(\gamma^{2}-1\right) \frac{a_{l}^{2}}{2 g} \geq z^{\#}-z_{l}, \\
h_{l}^{\#}=\left(h_{l}+\left(\gamma^{2}-1\right) \frac{a_{l}^{2}}{2 g}+z_{l}-z^{\#}\right)_{+} \text {otherwise }
\end{array}\right.
$$

and (3.15) is equivalent for $h_{r}>0$ to

$$
\left\{\begin{array}{l}
h_{r}^{\#}-\frac{(h a)_{r}^{2}}{2 g\left(h_{r}^{\#}\right)^{2}}=h_{r}-\frac{a_{r}^{2}}{2 g}+z_{r}-z^{\#}, \quad \text { if }\left(1-\frac{1}{\gamma}\right) h_{r}+\left(\gamma^{2}-1\right) \frac{a_{r}^{2}}{2 g} \geq z^{\#}-z_{r}, \\
h_{r}^{\#}=\left(h_{r}+\left(\gamma^{2}-1\right) \frac{a_{r}^{2}}{2 g}+z_{r}-z^{\#}\right)_{+} \text {otherwise. }
\end{array}\right.
$$

In practice we solve the equation on $h_{l}^{\#}$ in the first line of (3.17) (respectively $h_{r}^{\#}$ in the first line of (3.18)) by Newton's method starting with the initial guess 
$\max \left(h_{l}+z_{l}-z^{\#}, h_{l} / \gamma\right)\left(\right.$ respectively $\left.\max \left(h_{r}+z_{r}-z^{\#}, h_{r} / \gamma\right)\right)$. Then the iterative method converges increasingly to $h_{l}^{\#}$ (respectively $h_{r}^{\#}$ ).

We define then

$$
a_{l}^{\#}=\kappa_{l} a_{l}, \quad a_{r}^{\#}=\kappa_{r} a_{r},
$$

with

$$
\kappa_{l}=\min \left(\frac{h_{l}}{h_{l}^{\#}}, \gamma\right), \quad \kappa_{r}=\min \left(\frac{h_{r}}{h_{r}^{\#}}, \gamma\right),
$$

(we set $\kappa_{l}=1$ if $h_{l}=0, \kappa_{r}=1$ if $h_{r}=0$ ), and

$$
U_{l}^{\#}=\left(h_{l}^{\#}, h_{l}^{\#} u_{l}, h_{l}^{\#} v_{l}, h_{l}^{\#} a_{l}^{\#}, h_{l}^{\#} b_{l}\right), \quad U_{r}^{\#}=\left(h_{r}^{\#}, h_{r}^{\#} u_{r}, h_{r}^{\#} v_{r}, h_{r}^{\#} a_{r}^{\#}, h_{r}^{\#} b_{r}\right) .
$$

The left and right numerical fluxes are finally defined by

$$
\begin{gathered}
F_{l}\left(U_{l}, U_{r}, \Delta z\right)=\mathcal{F}_{l}\left(U_{l}^{\#}, U_{r}^{\#}\right) \\
+\left(0, g \frac{h_{l}^{2}}{2}-h_{l} a_{l}^{2}-g \frac{h_{l}^{\# 2}}{2}+\kappa_{l} h_{l} a_{l}^{2}, 0,\right. \\
\left.\kappa_{l}\left((h a)_{l}^{\#}-(h a)_{l}\right) u_{l},\left((h a)_{l}^{\#}-(h a)_{l}\right) v_{l}\right) \\
+\left(\kappa_{l}-1\right)\left(0,0,0, \mathcal{F}_{l}^{h a}\left(U_{l}^{\#}, U_{r}^{\#}\right), 0\right), \\
F_{r}\left(U_{l}, U_{r}, \Delta z\right)=\mathcal{F}_{r}\left(U_{l}^{\#}, U_{r}^{\#}\right) \\
+\left(0, g \frac{h_{r}^{2}}{2}-h_{r} a_{r}^{2}-g \frac{h_{r}^{\# 2}}{2}+\kappa_{r} h_{r} a_{r}^{2}, 0,\right. \\
\left.\quad \kappa_{r}\left((h a)_{r}^{\#}-(h a)_{r}\right) u_{r},\left((h a)_{r}^{\#}-(h a)_{r}\right) v_{r}\right) \\
+\left(\kappa_{r}-1\right)\left(0,0,0, \mathcal{F}_{r}^{h a}\left(U_{l}^{\#}, U_{r}^{\#}\right), 0\right),
\end{gathered}
$$

where $\mathcal{F}_{l}$ and $\mathcal{F}_{r}$ are the numerical fluxes of [10] associated to the problem without topography, and $\mathcal{F}^{h}$ is its common left/right height flux. We have again the extension property

$$
z_{l}=z_{r} \text { implies }\left\{\begin{array}{l}
U_{l}^{\#}=U_{l}, U_{r}^{\#}=U_{r}, \\
F_{l}\left(U_{l}, U_{r}, 0\right)=\mathcal{F}_{l}\left(U_{l}, U_{r}\right), F_{r}\left(U_{l}, U_{r}, 0\right)=\mathcal{F}_{r}\left(U_{l}, U_{r}\right) .
\end{array}\right.
$$

Theorem 3.2. The scheme (3.1) with the numerical fluxes $F_{l}, F_{r}$ defined by (3.22), (3.23) with the reconstruction (3.13)-(3.15), (3.19)-(3.21) satisfies the following properties.

(i) It is conservative in the variables $h$ and $h v$,

(ii) It is consistent with (1.5)-(1.9) for smooth solutions,

(iii) It keeps the positivity of $h$ under the CFL condition $\Delta t A\left(U_{l}^{\#}, U_{r}^{\#}\right) \leq$ $\frac{1}{2} \min \left(\Delta x_{l}, \Delta x_{r}\right)$ with $A(. .$.$) the maximum speed of the homogeneous solver,$ defined by [10, eq. (4.8)],

(iv) It satisfies a semi-discrete energy inequality associated to (1.11),

(v) It is well-balanced with respect to steady material and Alfven contact discontinuities without jump in topography, 
(vi) It is well-balanced with respect to the steady states (2.6) corresponding to material and Alfven resonance.

(vii) It is well-balanced with respect to the steady states (2.4) that satisfy

$$
\max \left(\frac{h_{l}}{h_{r}}, \frac{h_{r}}{h_{l}}\right) \leq \gamma
$$

(viii) The relation $h a=c s t$ is preserved by the scheme provided that at each interface the data satisfy

$$
\max \left(\frac{h_{l}}{h_{l}^{\#}}, \frac{h_{r}}{h_{r}^{\#}}\right) \leq \gamma \quad \text { whenever } h_{l}>0 \text { and } h_{r}>0 .
$$

As in the scheme of Theorem 3.1, the parameter $\gamma \geq 1$ is present here to remove the singularity of dividing by $h_{l}^{\#}$ and $h_{r}^{\#}$ in (3.20). In practice the choice of $\gamma$ is made by taking it large enough to include a large set of data that will satisfy (3.25) and (3.26), but not too large otherwise it would lead to eventually large values of $\kappa_{l}, \kappa_{r}$. The choice $\gamma=1$ is nevertheless possible, it only removes the properties (vii) and (viii) since they reduce to trivial states. Note that for $\gamma=1$, the schemes of Theorems 3.1 and 3.2 indeed coincide.

\section{Proof of the main results}

This section is devoted to the proof of the main results Theorems 3.1 and 3.2.

\subsection{Proof of Theorem 3.1}

The proof of (i), i.e. $F_{l}^{h}=F_{r}^{h}, F_{l}^{h v}=F_{r}^{h v}$, is obvious from formulas (3.8), (3.9) since the homogeneous solver already satisfies this property. We omit the proof of (iii), which follows the proof of Proposition 4.14 in [8].

The property (v) is inherited from the homogeneous solver that is described in [10], according to (3.10). We recall more explicitly that, defining

$$
F(U)=\left(h u, h u^{2}+P, h u v+P_{\perp}, 0, h b u-h a v\right)
$$

with $P$ and $P_{\perp}$ defined by (1.10), this property of well-balancing for the homogeneous solver means that $\mathcal{F}_{l}\left(U_{l}, U_{r}\right)=F\left(U_{l}\right)$ and $\mathcal{F}_{r}\left(U_{l}, U_{r}\right)=F\left(U_{r}\right)$ for all data of the form:

$$
u_{l}=u_{r}=0, v_{l}=v_{r}, P\left(U_{l}\right)=P\left(U_{r}\right), P_{\perp}\left(U_{l}\right)=P_{\perp}\left(U_{r}\right),
$$

or

$$
h_{l}=h_{r}, a_{l}=a_{r} \neq 0, u_{l}=u_{r}=\left|a_{l}\right|, b_{l} \operatorname{sgn}\left(a_{l}\right)-v_{l}=b_{r} \operatorname{sgn}\left(a_{r}\right)-v_{r},
$$

or

$$
h_{l}=h_{r}, a_{l}=a_{r} \neq 0, u_{l}=u_{r}=-\left|a_{l}\right|, b_{l} \operatorname{sgn}\left(a_{l}\right)+v_{l}=b_{r} \operatorname{sgn}\left(a_{r}\right)+v_{r},
$$

or

$$
h_{l}=h_{r}, u_{l}=u_{r}=0, a_{l}=a_{r}=0 .
$$


For the proof of (vi), consider data $U_{l}, U_{r}, z_{l}, z_{r}$ satisfying (2.6), i.e. $u_{l}=u_{r}=0$, $h_{l}+z_{l}=h_{r}+z_{r}, a_{l}=a_{r}=0$. Then we get $h_{l}^{\#}=h_{r}^{\#}, a_{l}^{\#}=a_{r}^{\#}=0$, and the fluxes $\mathcal{F}_{l}, \mathcal{F}_{r}$ are evaluated on states $U_{l}^{\#}, U_{r}^{\#}$ of the type (4.5). Thus $\mathcal{F}_{l}\left(U_{l}^{\#}, U_{r}^{\#}\right)=F\left(U_{l}^{\#}\right)$ and $\mathcal{F}_{r}\left(U_{l}^{\#}, U_{r}^{\#}\right)=F\left(U_{r}^{\#}\right)$. Using the form (4.1) of $F$ with $u=a=0$ and plugging this in (3.8), (3.9) we obtain $F_{l}=F\left(U_{l}\right), F_{r}=F\left(U_{r}\right)$, which proves the claim.

For the proof of (vii), consider data $U_{l}, U_{r}, z_{l}, z_{r}$ satisfying (2.3), i.e. $u_{l}=$ $u_{r}=0, v_{l}=v_{r}, h_{l}+z_{l}=h_{r}+z_{r}, \sqrt{h_{l}} a_{l}=\sqrt{h_{r}} a_{r} \neq 0, \sqrt{h_{l}} b_{l}=\sqrt{h_{r}} b_{r}$. Then from (3.3) we get

$$
h_{l}^{\#}=h_{r}^{\#} \equiv h^{\#},
$$

the common value $h^{\#}$ being $h_{r}$ if $\Delta z \geq 0$, or $h_{l}$ if $\Delta z \leq 0$. Using condition (3.11), according to (3.4), (3.5), (3.6), we get $\kappa_{l}=\sqrt{h_{l} / h_{l}^{\#}}, \kappa_{r}=\sqrt{h_{r} / h_{r}^{\#}}$, $\sqrt{h_{l}^{\#}} a_{l}^{\#}=\sqrt{h_{l}} a_{l}, \sqrt{h_{r}^{\#}} a_{r}^{\#}=\sqrt{h_{r}} a_{r}, \sqrt{h_{l}^{\#}} b_{l}^{\#}=\sqrt{h_{l}} b_{l}, \sqrt{h_{r}^{\#}} b_{r}^{\#}=\sqrt{h_{r}} b_{r}$. Thus

$$
\sqrt{h_{l}^{\#}} a_{l}^{\#}=\sqrt{h_{r}^{\#}} a_{r}^{\#}, \quad \sqrt{h_{l}^{\#}} b_{l}^{\#}=\sqrt{h_{r}^{\#}} b_{r}^{\#} .
$$

Using (4.6), (4.7), we get

$$
U_{l}^{\#}=U_{r}^{\#} \equiv U^{\#} \equiv\left(h^{\#}, 0, h^{\#} v^{\#}, h^{\#} a^{\#}, h^{\#} b^{\#}\right) .
$$

We observe that then $\mathcal{F}_{l}\left(U_{l}^{\#}, U_{r}^{\#}\right)=\mathcal{F}_{r}\left(U_{l}^{\#}, U_{r}^{\#}\right)=F\left(U^{\#}\right)$, and that indeed

$$
F\left(U^{\#}\right)=\left(0, g\left(h^{\#}\right)^{2} / 2-h^{\#}\left(a^{\#}\right)^{2},-h^{\#} a^{\#} b^{\#}, 0,-h^{\#} a^{\#} v^{\#}\right) .
$$

The formulas (3.8), (3.9) yield

$$
\begin{gathered}
F_{l}=\left(0, g h_{l}^{2} / 2-h_{l} a_{l}^{2},-h_{l} a_{l} b_{l}, 0,-h_{l} a_{l} v_{l}\right)=F\left(U_{l}\right), \\
F_{r}=\left(0, g h_{r}^{2} / 2-h_{r} a_{r}^{2},-h_{r} a_{r} b_{r}, 0,-h_{r} a_{r} v_{r}\right)=F\left(U_{r}\right),
\end{gathered}
$$

which proves the claim.

\subsubsection{Consistency in Theorem 3.1}

In order to get (ii) in Theorem 3.1 in the sense of Definition 4.2 in [8], we need to prove that

$$
F_{l}(U, U, 0)=F_{r}(U, U, 0)=F(U)
$$

and that as $U_{l} \rightarrow U, U_{r} \rightarrow U, \Delta z \rightarrow 0$,

$$
\begin{gathered}
F_{r}\left(U_{l}, U_{r}, \Delta z\right)-F_{l}\left(U_{l}, U_{r}, \Delta z\right)=-B(u, v)\left((h a)_{r}-(h a)_{l}\right) \\
\quad+(0,-g h \Delta z, 0,0,0)+o\left(\left|U_{l}-U\right|+\left|U_{r}-U\right|+|\Delta z|\right),
\end{gathered}
$$

with

$$
B(u, v)=(0,0,0, u, v) .
$$


The consistency with the exact flux (4.12) is obviously satisfied because of the property (3.10). In order to prove the consistency with the source (4.13), we write

$$
\begin{aligned}
& F_{r}\left(U_{l}, U_{r}, \Delta z\right)-F_{l}\left(U_{l}, U_{r}, \Delta z\right) \\
= & \mathcal{F}_{r}\left(U_{l}^{\#}, U_{r}^{\#}\right)-\mathcal{F}_{l}\left(U_{l}^{\#}, U_{r}^{\#}\right) \\
& +B\left(u_{r}, v_{r}\right)\left(\kappa_{r}(h a)_{r}^{\#}-(h a)_{r}\right)-B\left(u_{l}, v_{l}\right)\left(\kappa_{l}(h a)_{l}^{\#}-(h a)_{l}\right) \\
& +\left(\kappa_{r}-1\right)\left(0,0,0, \mathcal{F}_{r}^{h a}\left(U_{l}^{\#}, U_{r}^{\#}\right), \mathcal{F}_{r}^{h b}\left(U_{l}^{\#}, U_{r}^{\#}\right)\right) \\
& -\left(\kappa_{l}-1\right)\left(0,0,0, \mathcal{F}_{l}^{h a}\left(U_{l}^{\#}, U_{r}^{\#}\right), \mathcal{F}_{l}^{h b}\left(U_{l}^{\#}, U_{r}^{\#}\right)\right) \\
& +\mathcal{F}^{h}\left(U_{l}^{\#}, U_{r}^{\#}\right)\left(0,0,0, \frac{a_{r}}{2}\left(1-\kappa_{r}^{2}\right), \frac{b_{r}}{2}\left(1-\kappa_{r}^{2}\right)\right) \\
& -\mathcal{F}^{h}\left(U_{l}^{\#}, U_{r}^{\#}\right)\left(0,0,0, \frac{a_{l}}{2}\left(1-\kappa_{l}^{2}\right), \frac{b_{l}}{2}\left(1-\kappa_{l}^{2}\right)\right) \\
& +\left(0, g \frac{h_{l}^{\# 2}}{2}-g \frac{h_{l}^{2}}{2}+g \frac{h_{r}^{2}}{2}-g \frac{h_{r}^{\# 2}}{2}, 0,0,0\right) .
\end{aligned}
$$

Let us denote $\Delta=\left|U_{l}-U\right|+\left|U_{r}-U\right|+|\Delta z|$. When $U_{l}, U_{r} \rightarrow U$ and $\Delta z \rightarrow 0$ one has from (3.3)-(3.7) $\kappa_{l}-1=O(\Delta), \kappa_{r}-1=O(\Delta)$, and thus $U_{l}^{\#}-U=O(\Delta)$, $U_{r}^{\#}-U=O(\Delta)$ (we consider only the case $h>0$ here). Then the consistency of the numerical flux without source obtained in [10] gives

$$
\mathcal{F}_{r}\left(U_{l}^{\#}, U_{r}^{\#}\right)-\mathcal{F}_{l}\left(U_{l}^{\#}, U_{r}^{\#}\right)=-B(u, v)\left((h a)_{r}^{\#}-(h a)_{l}^{\#}\right)+o(\Delta) .
$$

Next, we have

$$
B\left(u_{r}, v_{r}\right)\left(\kappa_{r}(h a)_{r}^{\#}-(h a)_{r}\right)=B(u, v)\left(\kappa_{r}(h a)_{r}^{\#}-(h a)_{r}\right)+o(\Delta),
$$

and

$$
B\left(u_{l}, v_{l}\right)\left(\kappa_{l}(h a)_{l}^{\#}-(h a)_{l}\right)=B(u, v)\left(\kappa_{l}(h a)_{l}^{\#}-(h a)_{l}\right)+o(\Delta) .
$$

Summing up (4.16), (4.17), (4.18), we obtain

$$
\begin{aligned}
& \mathcal{F}_{r}\left(U_{l}^{\#}, U_{r}^{\#}\right)-\mathcal{F}_{l}\left(U_{l}^{\#}, U_{r}^{\#}\right) \\
& +B\left(u_{r}, v_{r}\right)\left(\kappa_{r}(h a)_{r}^{\#}-(h a)_{r}\right)-B\left(u_{l}, v_{l}\right)\left(\kappa_{l}(h a)_{l}^{\#}-(h a)_{l}\right) \\
= & B(u, v)\left(\kappa_{r}-1\right)(h a)_{r}^{\#}-B(u, v)\left(\kappa_{l}-1\right)(h a)_{l}^{\#} \\
& -B(u, v)\left((h a)_{r}-(h a)_{l}\right)+o(\Delta) . \\
= & B(u, v)\left(\kappa_{r}-1\right)(h a)-B(u, v)\left(\kappa_{l}-1\right)(h a) \\
& -B(u, v)\left((h a)_{r}-(h a)_{l}\right)+o(\Delta) .
\end{aligned}
$$

Now we look at the terms in the right-hand side of (4.15) from the third to the sixth line. Using that $\mathcal{F}_{l}^{h a}(U, U)=\mathcal{F}_{r}^{h a}(U, U)=0$ and $\mathcal{F}_{l}^{h b}(U, U)=\mathcal{F}_{r}^{h b}(U, U)=$ $h b u$ - hav, we deduce

$$
\begin{aligned}
& \left(\kappa_{r}-1\right)\left(0,0,0, \mathcal{F}_{r}^{h a}\left(U_{l}^{\#}, U_{r}^{\#}\right), \mathcal{F}_{r}^{h b}\left(U_{l}^{\#}, U_{r}^{\#}\right)\right) \\
& =\left(\kappa_{r}-1\right)(0,0,0,0, h b u-h a v)+o(\Delta),
\end{aligned}
$$


and

$$
\begin{aligned}
& -\left(\kappa_{l}-1\right)\left(0,0,0, \mathcal{F}_{l}^{h a}\left(U_{l}^{\#}, U_{r}^{\#}\right), \mathcal{F}_{l}^{h b}\left(U_{l}^{\#}, U_{r}^{\#}\right)\right) \\
& =-\left(\kappa_{l}-1\right)(0,0,0,0, h b u-h a v)+o(\Delta) .
\end{aligned}
$$

Writing $1-\kappa_{r}^{2}=\left(1+\kappa_{r}\right)\left(1-\kappa_{r}\right)$, we get asymptotically

$$
\frac{a_{r}}{2}\left(1-\kappa_{r}^{2}\right)=a\left(1-\kappa_{r}\right)+o(\Delta) .
$$

Similarly, we have

$$
\begin{aligned}
& \frac{a_{l}}{2}\left(1-\kappa_{l}^{2}\right)=a\left(1-\kappa_{l}\right)+o(\Delta), \\
& \frac{b_{r}}{2}\left(1-\kappa_{r}^{2}\right)=b\left(1-\kappa_{r}\right)+o(\Delta), \\
& \frac{b_{l}}{2}\left(1-\kappa_{l}^{2}\right)=b\left(1-\kappa_{l}\right)+o(\Delta) .
\end{aligned}
$$

Using (4.22), (4.23), (4.24), (4.25) and the property $\mathcal{F}^{h}(U, U)=h u$, we obtain

$$
\begin{aligned}
& \mathcal{F}^{h}\left(U_{l}^{\#}, U_{r}^{\#}\right)\left(0,0,0, \frac{a_{r}}{2}\left(1-\kappa_{r}^{2}\right), \frac{b_{r}}{2}\left(1-\kappa_{r}^{2}\right)\right) \\
= & \left(0,0,0, \operatorname{hua}\left(1-\kappa_{r}\right), h u b\left(1-\kappa_{r}\right)\right)+o(\Delta), \\
& -\mathcal{F}^{h}\left(U_{l}^{\#}, U_{r}^{\#}\right)\left(0,0,0, \frac{a_{l}}{2}\left(1-\kappa_{l}^{2}\right), \frac{b_{l}}{2}\left(1-\kappa_{l}^{2}\right)\right) \\
= & -\left(0,0,0, \text { hua }\left(1-\kappa_{l}\right), h u b\left(1-\kappa_{l}\right)\right)+o(\Delta) .
\end{aligned}
$$

The sum of (4.20), (4.21), (4.26), (4.27) gives the asymptotic formula

$$
\begin{aligned}
& \left(\kappa_{r}-1\right)\left(0,0,0, \mathcal{F}_{r}^{h a}\left(U_{l}^{\#}, U_{r}^{\#}\right), \mathcal{F}_{r}^{h b}\left(U_{l}^{\#}, U_{r}^{\#}\right)\right) \\
& -\left(\kappa_{l}-1\right)\left(0,0,0, \mathcal{F}_{l}^{h a}\left(U_{l}^{\#}, U_{r}^{\#}\right), \mathcal{F}_{l}^{h b}\left(U_{l}^{\#}, U_{r}^{\#}\right)\right) \\
& +\mathcal{F}^{h}\left(U_{l}^{\#}, U_{r}^{\#}\right)\left(0,0,0, \frac{a_{r}}{2}\left(1-\kappa_{r}^{2}\right), \frac{b_{r}}{2}\left(1-\kappa_{r}^{2}\right)\right) \\
& -\mathcal{F}^{h}\left(U_{l}^{\#}, U_{r}^{\#}\right)\left(0,0,0, \frac{a_{l}}{2}\left(1-\kappa_{l}^{2}\right), \frac{b_{l}}{2}\left(1-\kappa_{l}^{2}\right)\right) \\
& =-B(u, v)\left(\kappa_{r}-1\right)(h a)+B(u, v)\left(\kappa_{l}-1\right)(h a)+o(\Delta) .
\end{aligned}
$$

Adding (4.19) and (4.28), we obtain the consistency of the nonconservative magnetic terms

$$
\begin{aligned}
& \mathcal{F}_{r}\left(U_{l}^{\#}, U_{r}^{\#}\right)-\mathcal{F}_{l}\left(U_{l}^{\#}, U_{r}^{\#}\right) \\
& +B\left(u_{r}, v_{r}\right)\left(\kappa_{r}(h a)_{r}^{\#}-(h a)_{r}\right)-B\left(u_{l}, v_{l}\right)\left(\kappa_{l}(h a)_{l}^{\#}-(h a)_{l}\right) \\
& +\left(\kappa_{r}-1\right)\left(0,0,0, \mathcal{F}_{r}^{h a}\left(U_{l}^{\#}, U_{r}^{\#}\right), \mathcal{F}_{r}^{h b}\left(U_{l}^{\#}, U_{r}^{\#}\right)\right) \\
& -\left(\kappa_{l}-1\right)\left(0,0,0, \mathcal{F}_{l}^{h a}\left(U_{l}^{\#}, U_{r}^{\#}\right), \mathcal{F}_{l}^{h b}\left(U_{l}^{\#}, U_{r}^{\#}\right)\right) \\
& +\mathcal{F}^{h}\left(U_{l}^{\#}, U_{r}^{\#}\right)\left(0,0,0, \frac{a_{r}}{2}\left(1-\kappa_{r}^{2}\right), \frac{b_{r}}{2}\left(1-\kappa_{r}^{2}\right)\right) \\
& -\mathcal{F}^{h}\left(U_{l}^{\#}, U_{r}^{\#}\right)\left(0,0,0, \frac{a_{l}}{2}\left(1-\kappa_{l}^{2}\right), \frac{b_{l}}{2}\left(1-\kappa_{l}^{2}\right)\right) \\
& =-B(u, v)\left((h a)_{r}-(h a)_{l}\right)+o(\Delta) .
\end{aligned}
$$


Finally, as in the unmodified hydrostatic reconstruction scheme, the last line in (4.15) gives the nonconservative topography term

$$
\left(0, g \frac{h_{l}^{\# 2}}{2}-g \frac{h_{l}^{2}}{2}+g \frac{h_{r}^{2}}{2}-g \frac{h_{r}^{\# 2}}{2}, 0,0,0\right)=(0,-g h \Delta z, 0,0,0)+o(\Delta) .
$$

With (4.29), all the terms in (4.15) have been expanded, and we get (4.13).

\subsubsection{Entropy inequality in Theorem 3.1}

Let us finally prove the property (iv) in Theorem 3.1. At the continuous level, the energy inequality (1.11) can be written

$$
\partial_{t} \tilde{E}+\partial_{x} \tilde{G} \leq 0,
$$

with

$$
\tilde{E}(U, z)=E(U)+g h z, \quad \tilde{G}(U, z)=G(U)+g h z u,
$$

and

$$
\begin{gathered}
E(U)=\frac{1}{2} h\left(u^{2}+v^{2}\right)+\frac{1}{2} g h^{2}+\frac{1}{2} h\left(a^{2}+b^{2}\right), \\
G(U)=E(U) u+P(U) u+\stackrel{P}{\perp}_{\perp}(U) v .
\end{gathered}
$$

As before, $U=(h, h u, h v, h a, h b)$ and $P, P_{\perp}$ are defined by (1.10). As proved in [10], the scheme without topography satisfies a fully discrete energy inequality. According to $[8$, section 2.2.2], it implies that it satisfies also a semi-discrete energy inequality, under the form

$$
\begin{gathered}
G\left(U_{r}\right)+E^{\prime}\left(U_{r}\right)\left(\mathcal{F}_{r}\left(U_{l}, U_{r}\right)-F\left(U_{r}\right)\right) \leq \mathcal{G}\left(U_{l}, U_{r}\right), \\
\mathcal{G}\left(U_{l}, U_{r}\right) \leq G\left(U_{l}\right)+E^{\prime}\left(U_{l}\right)\left(\mathcal{F}_{l}\left(U_{l}, U_{r}\right)-F\left(U_{l}\right)\right),
\end{gathered}
$$

for all values of $U_{l}, U_{r}$, where $E^{\prime}$ is the derivative of $E$ with respect to $U, F$ is defined in (4.1), and $\mathcal{G}\left(U_{l}, U_{r}\right)$ is a consistent energy flux.

Then, for the scheme with topography, the characterization of the semidiscrete energy inequality writes

$$
\begin{gathered}
\tilde{G}\left(U_{r}, z_{r}\right)+\tilde{E}^{\prime}\left(U_{r}, z_{r}\right)\left(F_{r}-F\left(U_{r}\right)\right) \leq \tilde{\mathcal{G}}\left(U_{l}, U_{r}, z_{l}, z_{r}\right), \\
\tilde{\mathcal{G}}\left(U_{l}, U_{r}, z_{l}, z_{r}\right) \leq \tilde{G}\left(U_{l}, z_{l}\right)+\tilde{E}^{\prime}\left(U_{l}, z_{l}\right)\left(F_{l}-F\left(U_{l}\right)\right),
\end{gathered}
$$

where $\tilde{E}$ and $\tilde{G}$ are defined by (4.32), $\tilde{E}^{\prime}$ is the derivative of $\tilde{E}$ with respect to $U$, and $\tilde{\mathcal{G}}$ is an unknown consistent numerical energy flux. Let us choose

$$
\tilde{\mathcal{G}}\left(U_{l}, U_{r}, z_{l}, z_{r}\right)=\mathcal{G}\left(U_{l}^{\#}, U_{r}^{\#}\right)+\mathcal{F}^{h}\left(U_{l}^{\#}, U_{r}^{\#}\right) g z^{\#},
$$

where $\mathcal{F}^{h}$ is the common h-component of $\mathcal{F}_{l}$ and $\mathcal{F}_{r}$, and for some $z^{\#}$ that is defined below. Then, noticing that $\tilde{E}^{\prime}(U, z)=E^{\prime}(U)+g z(1,0,0,0,0)$, we can write the desired inequalities (4.35) as

$$
\begin{aligned}
& G\left(U_{r}\right)+E^{\prime}\left(U_{r}\right)\left(F_{r}-F\left(U_{r}\right)\right)+\mathcal{F}^{h}\left(U_{l}^{\#}, U_{r}^{\#}\right) g z_{r} \\
\leq & \mathcal{G}\left(U_{l}^{\#}, U_{r}^{\#}\right)+\mathcal{F}^{h}\left(U_{l}^{\#}, U_{r}^{\#}\right) g z^{\#}, \\
& \mathcal{G}\left(U_{l}^{\#}, U_{r}^{\#}\right)+\mathcal{F}^{h}\left(U_{l}^{\#}, U_{r}^{\#}\right) g z^{\#} \\
\leq & G\left(U_{l}\right)+E^{\prime}\left(U_{l}\right)\left(F_{l}-F\left(U_{l}\right)\right)+\mathcal{F}^{h}\left(U_{l}^{\#}, U_{r}^{\#}\right) g z_{l} .
\end{aligned}
$$


By using (4.34) evaluated at $U_{l}^{\#}, U_{r}^{\#}$ and comparing the result with (4.37) and (4.38), we get the sufficient conditions

$$
\begin{aligned}
& G\left(U_{r}\right)+E^{\prime}\left(U_{r}\right)\left(F_{r}-F\left(U_{r}\right)\right)+\mathcal{F}^{h}\left(U_{l}^{\#}, U_{r}^{\#}\right) g z_{r} \\
\leq & G\left(U_{r}^{\#}\right)+E^{\prime}\left(U_{r}^{\#}\right)\left(\mathcal{F}_{r}\left(U_{l}^{\#}, U_{r}^{\#}\right)-F\left(U_{r}^{\#}\right)\right)+\mathcal{F}^{h}\left(U_{l}^{\#}, U_{r}^{\#}\right) g z^{\#}, \\
& G\left(U_{l}^{\#}\right)+E^{\prime}\left(U_{l}^{\#}\right)\left(\mathcal{F}_{l}\left(U_{l}^{\#}, U_{r}^{\#}\right)-F\left(U_{l}^{\#}\right)\right)+\mathcal{F}^{h}\left(U_{l}^{\#}, U_{r}^{\#}\right) g z^{\#} \\
\leq & G\left(U_{l}\right)+E^{\prime}\left(U_{l}\right)\left(F_{l}-F\left(U_{l}\right)\right)+\mathcal{F}^{h}\left(U_{l}^{\#}, U_{r}^{\#}\right) g z_{l} .
\end{aligned}
$$

Let us focus on (4.39), that can be rewritten as

$$
\begin{aligned}
{\left[G-E^{\prime} F\right]_{r \#}^{r}+E^{\prime}\left(U_{r}\right) F_{r}-E^{\prime}\left(U_{r}^{\#}\right) \mathcal{F}_{r}\left(U_{l}^{\#}, U_{r}^{\#}\right) } & \\
+ & g\left(z_{r}-z^{\#}\right) \mathcal{F}^{h}\left(U_{l}^{\#}, U_{r}^{\#}\right) \leq 0,
\end{aligned}
$$

with

$$
\left[G-E^{\prime} F\right]_{r \#}^{r} \equiv\left(G\left(U_{r}\right)-E^{\prime}\left(U_{r}\right) F\left(U_{r}\right)\right)-\left(G\left(U_{r}^{\#}\right)-E^{\prime}\left(U_{r}^{\#}\right) F\left(U_{r}^{\#}\right)\right) .
$$

We compute now

$$
E^{\prime}(U)=\left(-\left(u^{2}+v^{2}\right) / 2+g h-\left(a^{2}+b^{2}\right) / 2, u, v, a, b\right),
$$

and using (4.33), (4.1), we deduce the identity

$$
G(U)-E^{\prime}(U) F(U)=-g \frac{h^{2}}{2} u+h a(a u+b v)=-P(U) u-P_{\perp}(U) v .
$$

Then, according to the definition (3.9) of $F_{r}$,

$$
\begin{aligned}
E^{\prime}\left(U_{r}\right) F_{r}= & E^{\prime}\left(U_{r}\right) \mathcal{F}_{r}\left(U_{l}^{\#}, U_{r}^{\#}\right) \\
+ & E^{\prime}\left(U_{r}\right)\left(0, g \frac{h_{r}^{2}}{2}-g \frac{h_{r}^{\# 2}}{2}, 0,\right. \\
& \left.\quad\left(\kappa_{r}(h a)_{r}^{\#}-(h a)_{r}\right) u_{r},\left(\kappa_{r}(h a)_{r}^{\#}-(h a)_{r}\right) v_{r}\right)+Q_{r},
\end{aligned}
$$

with

$$
\begin{aligned}
Q_{r}= & E^{\prime}\left(U_{r}\right)\left(\kappa_{r}-1\right)\left(0,0,0, \mathcal{F}_{r}^{h a}\left(U_{l}^{\#}, U_{r}^{\#}\right), \mathcal{F}_{r}^{h b}\left(U_{l}^{\#}, U_{r}^{\#}\right)\right) \\
& +E^{\prime}\left(U_{r}\right) \mathcal{F}^{h}\left(U_{l}^{\#}, U_{r}^{\#}\right)\left(0,0,0, \frac{a_{r}}{2}\left(1-\kappa_{r}^{2}\right), \frac{b_{r}}{2}\left(1-\kappa_{r}^{2}\right)\right) .
\end{aligned}
$$

Using (4.43) and (4.44), we can rewrite (4.45) as

$$
E^{\prime}\left(U_{r}\right) F_{r}=E^{\prime}\left(U_{r}\right) \mathcal{F}_{r}\left(U_{l}^{\#}, U_{r}^{\#}\right)-\left[G-E^{\prime} F\right]_{r \#}^{r}+Q_{r} .
$$

Thus the required inequality (4.41) simplifies to

$$
\left(E^{\prime}\left(U_{r}\right)-E^{\prime}\left(U_{r}^{\#}\right)\right) \mathcal{F}_{r}\left(U_{l}^{\#}, U_{r}^{\#}\right)+Q_{r}+g\left(z_{r}-z^{\#}\right) \mathcal{F}^{h}\left(U_{l}^{\#}, U_{r}^{\#}\right) \leq 0 .
$$


Now, one the one side, one can compute

$$
\begin{aligned}
Q_{r}= & \left(\kappa_{r}-1\right) a_{r} \mathcal{F}_{r}^{h a}\left(U_{l}^{\#}, U_{r}^{\#}\right)+\left(\kappa_{r}-1\right) b_{r} \mathcal{F}_{r}^{h b}\left(U_{l}^{\#}, U_{r}^{\#}\right) \\
& +\left(1-\kappa_{r}^{2}\right) \mathcal{F}^{h}\left(U_{l}^{\#}, U_{r}^{\#}\right) \frac{a_{r}^{2}+b_{r}^{2}}{2}
\end{aligned}
$$

On the other side, according to (4.43), we have

$$
\begin{aligned}
& E^{\prime}\left(U_{r}\right)-E^{\prime}\left(U_{r}^{\#}\right) \\
= & \left(g\left(h_{r}-h_{r}^{\#}\right)-\frac{a_{r}^{2}+b_{r}^{2}}{2}+\frac{\left(a_{r}^{\#}\right)^{2}+\left(b_{r}^{\#}\right)^{2}}{2}, 0,0, a_{r}-a_{r}^{\#}, b_{r}-b_{r}^{\#}\right) \\
= & \left(g\left(h_{r}-h_{r}^{\#}\right)-\left(1-\kappa_{r}^{2}\right) \frac{a_{r}^{2}+b_{r}^{2}}{2}, 0,0,\left(1-\kappa_{r}\right) a_{r},\left(1-\kappa_{r}\right) b_{r}\right) .
\end{aligned}
$$

Using both (4.49) and (4.50), we get

$$
\left(E^{\prime}\left(U_{r}\right)-E^{\prime}\left(U_{r}^{\#}\right)\right) \mathcal{F}_{r}\left(U_{l}^{\#}, U_{r}^{\#}\right)+Q_{r}=g\left(h_{r}-h_{r}^{\#}\right) \mathcal{F}^{h}\left(U_{l}^{\#}, U_{r}^{\#}\right) .
$$

Plugging this in (4.48), we obtain the sufficient right inequality

$$
g\left(h_{r}-h_{r}^{\#}+z_{r}-z^{\#}\right) \mathcal{F}^{h}\left(U_{l}^{\#}, U_{r}^{\#}\right) \leq 0 .
$$

A symmetric analysis for the left inequality (4.40) gives similarly

$$
g\left(h_{l}-h_{l}^{\#}+z_{l}-z^{\#}\right) \mathcal{F}^{h}\left(U_{l}^{\#}, U_{r}^{\#}\right) \geq 0 .
$$

We choose $z^{\#}=\max \left(z_{l}, z_{r}\right)$, so that (4.52), (4.53) can be finally put under the form

$$
\begin{gathered}
g\left(h_{r}-h_{r}^{\#}-(-\Delta z)_{+}\right) \mathcal{F}^{h}\left(U_{l}^{\#}, U_{r}^{\#}\right) \leq 0, \\
g\left(h_{l}-h_{l}^{\#}-(\Delta z)_{+}\right) \mathcal{F}^{h}\left(U_{l}^{\#}, U_{r}^{\#}\right) \geq 0 .
\end{gathered}
$$

Taking into account (3.3), we observe that if $h_{l}-(\Delta z)_{+} \geq 0$ then the second line of (4.54) is trivial. Otherwise $h_{l}^{\#}=0$ and the second inequality of (4.54) holds because $\mathcal{F}^{h}\left(0, U_{r}^{\#}\right) \leq 0$ by the $h$-nonnegativity of the numerical flux. The same argument is valid for the first inequality of (4.54), which concludes the proof of Theorem 3.1 .

\subsection{Proof of Theorem 3.2}

The proof of (i), i.e. $F_{l}^{h}=F_{r}^{h}, F_{l}^{h v}=F_{r}^{h v}$, is again obvious from formulas (3.22), (3.23) since the homogeneous solver already satisfies this property. The proof of (iii) follows the proof of Proposition 4.14 in [8], taking into account (3.16). The property (v) is inherited from the homogeneous solver that is described in [10], according to (3.24). The proof of (vi) concerning data of the form (2.6) is identical to that of Theorem 3.1 in Subsection 4.1.

For the proof of the specific well-balanced property (vii), consider data $U_{l}$, $U_{r}, z_{l}, z_{r}$ satisfying (2.4), i.e. $u_{l}=u_{r}=0, v_{l}=v_{r} \equiv v, h_{l}-\frac{a_{l}^{2}}{2 g}+z_{l}=h_{r}-\frac{a_{r}^{2}}{2 g}+z_{r}$, $h_{l} a_{l}=h_{r} a_{r} \neq 0, b_{l}=b_{r} \equiv b$. According to the assumption (3.25), from (3.13), (3.15) we get

$$
h_{l}^{\#}=h_{r}^{\#} \equiv h^{\#} \text {, }
$$


the common value $h^{\#}$ being $h_{r}$ if $z_{r}-z_{l} \geq 0$, or $h_{l}$ if $z_{r}-z_{l} \leq 0$. Then (3.19), (3.20) yield $\kappa_{l}=h_{l} / h_{l}^{\#}, \kappa_{r}=h_{r} / h_{r}^{\#}, h_{l}^{\#} a_{l}^{\#}=h_{l} a_{l}, h_{r}^{\#} a_{r}^{\#}=h_{r} a_{r}$. Thus

$$
h_{l}^{\#} a_{l}^{\#}=h_{r}^{\#} a_{r}^{\#} \neq 0
$$

and using (4.55) we obtain $a_{l}^{\#}=a_{r}^{\#} \equiv a^{\#}$. Then (3.21) yields

$$
U_{l}^{\#}=U_{r}^{\#} \equiv U^{\#} \equiv\left(h^{\#}, 0, h^{\#} v, h^{\#} a^{\#}, h^{\#} b\right) .
$$

We observe that then $\mathcal{F}_{l}\left(U_{l}^{\#}, U_{r}^{\#}\right)=\mathcal{F}_{r}\left(U_{l}^{\#}, U_{r}^{\#}\right)=F\left(U^{\#}\right)$, and that indeed

$$
F\left(U^{\#}\right)=\left(0, g\left(h^{\#}\right)^{2} / 2-h^{\#}\left(a^{\#}\right)^{2},-(h a)^{\#} b, 0,-(h a)^{\#} v\right) .
$$

The formulas (3.22), (3.23), finally give

$$
\begin{gathered}
F_{l}=\left(0, g h_{l}^{2} / 2-h_{l} a_{l}^{2},-h_{l} a_{l} b_{l}, 0,-h_{l} a_{l} v_{l}\right)=F\left(U_{l}\right), \\
F_{r}=\left(0, g h_{r}^{2} / 2-h_{r} a_{r}^{2},-h_{r} a_{r} b_{r}, 0,-h_{r} a_{r} v_{r}\right)=F\left(U_{r}\right),
\end{gathered}
$$

which proves the claim.

For proving (viii), consider data $U_{l}, U_{r}, z_{l}, z_{r}$ such that $h_{l} a_{l}=h_{r} a_{r}$. We have to prove that $F_{l}^{h a}\left(U_{l}, U_{r}, \Delta z\right)=F_{r}^{h a}\left(U_{l}, U_{r}, \Delta z\right)=0$, where the superscript $h a$ means that we take the $h a$ component of the numerical flux. We notice from [10, eq. (4.3)] that $h_{l} a_{l}=h_{r} a_{r}$ implies that $\mathcal{F}_{l}^{h a}\left(U_{l}, U_{r}\right)=\mathcal{F}_{r}^{h a}\left(U_{l}, U_{r}\right)=0$.

If $h_{l}=h_{r}=0$ then the result is trivial since $F_{l}=F_{r}=0$. If $h_{l}=0$ and $h_{r}>0$ then $a_{r}=0, h_{l}^{\#}=0, a_{r}^{\#}=0$, hence $h_{l}^{\#} a_{l}^{\#}=h_{r}^{\#} a_{r}^{\#}=0$. It follows that $\mathcal{F}_{l}^{h a}\left(U_{l}^{\#}, U_{r}^{\#}\right)=\mathcal{F}_{r}^{h a}\left(U_{l}^{\#}, U_{r}^{\#}\right)=0$, and the numerical flux formulas (3.22), (3.23) give $F_{l}^{h a}\left(U_{l}, U_{r}, \Delta z\right)=F_{r}^{h a}\left(U_{l}, U_{r}, \Delta z\right)=0$. The case $h_{r}=0$ and $h_{l}>0$ is similar.

We finally consider the case $h_{l}>0$ and $h_{r}>0$. According to the assumption (3.26), the formulas (3.20) give $\kappa_{l}=h_{l} / h_{l}^{\#}, \kappa_{r}=h_{r} / h_{r}^{\#}$. It follows with (3.19) that $h_{l}^{\#} a_{l}^{\#}=h_{l} a_{l}, h_{r}^{\#} a_{r}^{\#}=h_{r} a_{r}$. But since $h_{l} a_{l}=h_{r} a_{r}$, all these values of $h a$ are the same. We deduce that $\mathcal{F}_{l}^{h a}\left(U_{l}^{\#}, U_{r}^{\#}\right)=\mathcal{F}_{r}^{h a}\left(U_{l}^{\#}, U_{r}^{\#}\right)=0$, and the formulas (3.22), (3.23) give $F_{l}^{h a}\left(U_{l}, U_{r}, \Delta z\right)=F_{r}^{h a}\left(U_{l}, U_{r}, \Delta z\right)=0$.

\subsubsection{Consistency in Theorem 3.2}

In order to get the consistency (ii) in Theorem 3.2, in the sense of Definition 4.2 in [8], we need to prove that

$$
F_{l}(U, U, 0)=F_{r}(U, U, 0)=F(U),
$$

and that as $U_{l} \rightarrow U, U_{r} \rightarrow U, \Delta z \rightarrow 0$,

$$
\begin{aligned}
& F_{r}^{h u}\left(U_{l}, U_{r}, \Delta z\right)-F_{l}^{h u}\left(U_{l}, U_{r}, \Delta z\right)=-g h \Delta z+o(\Delta), \\
& F_{r}^{h a}\left(U_{l}, U_{r}, \Delta z\right)-F_{l}^{h a}\left(U_{l}, U_{r}, \Delta z\right)=-u\left((h a)_{r}-(h a)_{l}\right)+o(\Delta), \\
& F_{r}^{h b}\left(U_{l}, U_{r}, \Delta z\right)-F_{l}^{h b}\left(U_{l}, U_{r}, \Delta z\right)=-v\left((h a)_{r}-(h a)_{l}\right)+o(\Delta), \\
& \text { with } \Delta=\left|U_{l}-U\right|+\left|U_{r}-U\right|+|\Delta z| \text {. }
\end{aligned}
$$


The consistency with the exact flux (4.61) is obviously satisfied because of the property (3.24). In order to prove the consistency with the ha component of the source (4.63), we write

$$
\begin{aligned}
& F_{r}^{h a}\left(U_{l}, U_{r}, \Delta z\right)-F_{l}^{h a}\left(U_{l}, U_{r}, \Delta z\right) \\
= & \mathcal{F}_{r}^{h a}\left(U_{l}^{\#}, U_{r}^{\#}\right)-\mathcal{F}_{l}^{h a}\left(U_{l}^{\#}, U_{r}^{\#}\right) \\
& +\left(\kappa_{r}(h a)_{r}^{\#}-(h a)_{r}\right) u_{r}+\left((h a)_{r}-\kappa_{r} h_{r} a_{r}\right) u_{r} \\
& -\left(\kappa_{l}(h a)_{l}^{\#}-(h a)_{l}\right) u_{l}-\left((h a)_{l}-\kappa_{l} h_{l} a_{l}\right) u_{l} \\
& +\left(\kappa_{r}-1\right) \mathcal{F}_{r}^{h a}\left(U_{l}^{\#}, U_{r}^{\#}\right)-\left(\kappa_{l}-1\right) \mathcal{F}_{l}^{h a}\left(U_{l}^{\#}, U_{r}^{\#}\right) .
\end{aligned}
$$

When $U_{l}, U_{r} \rightarrow U$ and $\Delta z \rightarrow 0$ one has from (3.13)-(3.21) $\kappa_{l}-1=O(\Delta)$, $\kappa_{r}-1=O(\Delta)$, and thus $U_{l}^{\#}-U=O(\Delta), U_{r}^{\#}-U=O(\Delta)$ (we consider only the case $h>0$ here). Then the consistency of the numerical flux without source obtained in $[10]$ gives

$$
\mathcal{F}_{r}^{h a}\left(U_{l}^{\#}, U_{r}^{\#}\right)-\mathcal{F}_{l}^{h a}\left(U_{l}^{\#}, U_{r}^{\#}\right)=-u\left((h a)_{r}^{\#}-(h a)_{l}^{\#}\right)+o(\Delta) .
$$

Then

$$
\begin{aligned}
& \left(\kappa_{r}(h a)_{r}^{\#}-(h a)_{r}\right) u_{r}+\left((h a)_{r}-\kappa_{r} h_{r} a_{r}\right) u_{r} \\
= & \left(\kappa_{r}(h a)_{r}^{\#}-(h a)_{r}\right) u+h a u\left(1-\kappa_{r}\right)+o(\Delta), \\
& \left(\kappa_{l}(h a)_{l}^{\#}-(h a)_{l}\right) u_{l}+\left((h a)_{l}-\kappa_{l} h_{l} a_{l}\right) u_{l} \\
= & \left(\kappa_{l}(h a)_{l}^{\#}-(h a)_{l}\right) u+h a u\left(1-\kappa_{l}\right)+o(\Delta) .
\end{aligned}
$$

Using that $\mathcal{F}_{r}^{h a}(U, U)=0, \mathcal{F}_{l}^{h a}(U, U)=0$ we get

$$
\left(\kappa_{r}-1\right) \mathcal{F}_{r}^{h a}\left(U_{l}^{\#}, U_{r}^{\#}\right)=o(\Delta), \quad\left(\kappa_{l}-1\right) \mathcal{F}_{l}^{h a}\left(U_{l}^{\#}, U_{r}^{\#}\right)=o(\Delta) .
$$

From (4.65) using (4.66)-(4.69), we get

$$
\begin{aligned}
& F_{r}^{h a}\left(U_{l}, U_{r}, \Delta z\right)-F_{l}^{h a}\left(U_{l}, U_{r}, \Delta z\right) \\
= & -u\left((h a)_{r}^{\#}-(h a)_{l}^{\#}\right) \\
& +\left(\kappa_{r}(h a)_{r}^{\#}-(h a)_{r}\right) u+h a u\left(1-\kappa_{r}\right) \\
& -\left(\kappa_{l}(h a)_{l}^{\#}-(h a)_{l}\right) u-h a u\left(1-\kappa_{l}\right)+o(\Delta) .
\end{aligned}
$$

Then we deal with the terms with subscript $r$ and we compute

$$
\begin{aligned}
& -u(h a)_{r}^{\#}+\left(\kappa_{r}(h a)_{r}^{\#}-(h a)_{r}\right) u+h a u\left(1-\kappa_{r}\right) \\
= & -u(h a)_{r}+u\left(\kappa_{r}-1\right)(h a)_{r}^{\#}+\left(1-\kappa_{r}\right) h a u .
\end{aligned}
$$

In addition since $u\left(\kappa_{r}-1\right)(h a)_{r}^{\#}=h a u\left(\kappa_{r}-1\right)+o(\Delta)$ we get

$$
-u(h a)_{r}^{\#}+\left(\kappa_{r}(h a)_{r}^{\#}-(h a)_{r}\right) u+h a u\left(1-\kappa_{r}\right)=-u(h a)_{r}+o(\Delta) .
$$

We do a similar computation on the left side and we get

$$
u(h a)_{l}^{\#}-\left(\kappa_{l}(h a)_{l}^{\#}-(h a)_{l}\right) u-h a u\left(1-\kappa_{l}\right)=u(h a)_{l}+o(\Delta) .
$$


Finally we use (4.72) and (4.73) in (4.70) and we get (4.63).

In order to prove the consistency with the $h b$ component of the source (4.64), we write

$$
\begin{aligned}
& F_{r}^{h b}\left(U_{l}, U_{r}, \Delta z\right)-F_{l}^{h b}\left(U_{l}, U_{r}, \Delta z\right) \\
= & \mathcal{F}_{r}^{h b}\left(U_{l}^{\#}, U_{r}^{\#}\right)-\mathcal{F}_{l}^{h b}\left(U_{l}^{\#}, U_{r}^{\#}\right) \\
& +\left((h a)_{r}^{\#}-(h a)_{r}\right) v_{r}-\left((h a)_{l}^{\#}-(h a)_{l}\right) v_{l} .
\end{aligned}
$$

Then the consistency of the numerical flux without source obtained in [10] gives

$$
\mathcal{F}_{r}^{h b}\left(U_{l}^{\#}, U_{r}^{\#}\right)-\mathcal{F}_{l}^{h b}\left(U_{l}^{\#}, U_{r}^{\#}\right)=-v\left((h a)_{r}^{\#}-(h a)_{l}^{\#}\right)+o(\Delta) .
$$

Using this expansion in (4.74), we get (4.64).

In order to prove the consistency with the $h u$ component of the source (4.62), we write

$$
F_{r}^{h u}\left(U_{l}, U_{r}, \Delta z\right)-F_{l}^{h u}\left(U_{l}, U_{r}, \Delta z\right)=P_{r}\left(h_{r}\right)-P_{r}\left(h_{r}^{\#}\right)-P_{l}\left(h_{l}\right)+P_{l}\left(h_{l}^{\#}\right),
$$

with

$$
\begin{gathered}
P_{r}(h)=g \frac{h^{2}}{2}-h_{r} a_{r}^{2} \min \left(\frac{h_{r}}{h}, \gamma\right), \\
P_{l}(h)=g \frac{h^{2}}{2}-h_{l} a_{l}^{2} \min \left(\frac{h_{l}}{h}, \gamma\right) .
\end{gathered}
$$

We define

$$
\begin{gathered}
e_{r}(h)=\frac{g h}{2}+\frac{h_{r} a_{r}^{2}}{h} \min \left(\frac{h_{r}}{h}, \gamma\right)-\frac{a_{r}^{2}}{2} \min \left(\frac{h_{r}}{h}, \gamma\right)^{2}, \\
e_{l}(h)=\frac{g h}{2}+\frac{h_{l} a_{l}^{2}}{h} \min \left(\frac{h_{l}}{h}, \gamma\right)-\frac{a_{l}^{2}}{2} \min \left(\frac{h_{l}}{h}, \gamma\right)^{2} .
\end{gathered}
$$

They satisfy the relations $e_{l / r}^{\prime}=P_{l / r} / h^{2}$ which implies that $\left(e_{l / r}+P_{l / r} / h\right)^{\prime}=$ $P_{l / r}^{\prime} / h$. Using these identities we get

$$
P_{r}\left(h_{r}\right)-P_{r}\left(h_{r}^{\#}\right)=\left(\left(e_{r}+P_{r} / h\right)\left(h_{r}\right)-\left(e_{r}+P_{r} / h\right)\left(h_{r}^{\#}\right)\right) h_{r}^{\# \#}
$$

for some $h_{r}^{\# \#}$ between $h_{r}^{\#}$ and $h_{r}$, and

$$
P_{l}\left(h_{l}\right)-P_{l}\left(h_{l}^{\#}\right)=\left(\left(e_{l}+P_{l} / h\right)\left(h_{l}\right)-\left(e_{l}+P_{l} / h\right)\left(h_{l}^{\#}\right)\right) h_{l}^{\# \#}
$$

for some $h_{l}^{\# \#}$ between $h_{l}^{\#}$ and $h_{l}$. Moreover using (4.77), (4.78) we notice that (3.13), (3.15) are equivalent to

$$
\begin{aligned}
\left(e_{l}+P_{l} / h\right)\left(h_{l}^{\#}\right) & =\left(e_{l}+P_{l} / h\right)\left(h_{l}\right)+g\left(z_{l}-z^{\#}\right), \\
\left(e_{r}+P_{r} / h\right)\left(h_{r}^{\#}\right) & =\left(e_{r}+P_{r} / h\right)\left(h_{r}\right)+g\left(z_{r}-z^{\#}\right) .
\end{aligned}
$$

This is true indeed as soon as $h_{l}^{\#}>0, h_{r}^{\#}>0$, which holds for sufficiently small $\Delta$ since we assumed that $h>0$. Therefore we have $P_{l}\left(h_{l}\right)-P_{l}\left(h_{l}^{\#}\right)=$ $-g h_{l}^{\# \#}\left(z_{l}-z^{\#}\right), P_{r}\left(h_{r}\right)-P_{r}\left(h_{r}^{\#}\right)=-g h_{r}^{\# \#}\left(z_{r}-z^{\#}\right)$, and with (4.76) it gives $(4.62)$. 


\subsubsection{Entropy inequality in Theorem 3.2}

We here prove the property (iv) in Theorem 3.2. We start with the same preliminaries (4.31)-(4.44) of Subsubsection 4.1.2. Thus for the right side we have the sufficient entropy condition

$$
\begin{aligned}
{\left[G-E^{\prime} F\right]_{r \#}^{r}+E^{\prime}\left(U_{r}\right) F_{r}-E^{\prime}\left(U_{r}^{\#}\right) \mathcal{F}_{r}\left(U_{l}^{\#}, U_{r}^{\#}\right) } \\
+g\left(z_{r}-z^{\#}\right) \mathcal{F}^{h}\left(U_{l}^{\#}, U_{r}^{\#}\right) \leq 0 .
\end{aligned}
$$

Using the definition (3.23) of $F_{r}$ this can be rewritten

$$
\begin{aligned}
& {\left[G-E^{\prime} F\right]_{r \#}^{r}+E^{\prime}\left(U_{r}\right) C_{1}+E^{\prime}\left(U_{r}\right) C_{2}+E^{\prime}\left(U_{r}\right) C_{3}} \\
& +\left(E^{\prime}\left(U_{r}\right)-E^{\prime}\left(U_{r}^{\#}\right)\right) \mathcal{F}_{r}\left(U_{l}^{\#}, U_{r}^{\#}\right)+g\left(z_{r}-z^{\#}\right) \mathcal{F}^{h}\left(U_{l}^{\#}, U_{r}^{\#}\right) \leq 0,
\end{aligned}
$$

with

$$
\begin{gathered}
C_{1}=\left(0, g \frac{h_{r}^{2}}{2}-g \frac{h_{r}^{\# 2}}{2}, 0,\left(\kappa_{r}(h a)_{r}^{\#}-(h a)_{r}\right) u_{r},\left((h a)_{r}^{\#}-(h a)_{r}\right) v_{r}\right), \\
C_{2}=\left(0,-h_{r} a_{r}^{2}+\kappa_{r} h_{r} a_{r}^{2}, 0,\left((h a)_{r}-\kappa_{r} h_{r} a_{r}\right) u_{r}, 0\right) \\
C_{3}=\left(\kappa_{r}-1\right)\left(0,0,0, \mathcal{F}_{r}^{h a}\left(U_{l}^{\#}, U_{r}^{\#}\right), 0\right) .
\end{gathered}
$$

Using (4.43) and (4.44) we get with (4.84)

$$
\left[G-E^{\prime} F\right]_{r \#}^{r}+E^{\prime}\left(U_{r}\right) C_{1}=0 .
$$

Moreover using (4.43) we have

$$
E^{\prime}\left(U_{r}\right) C_{2}=0 .
$$

Thus the sufficient condition (4.83) reduces to

$$
\begin{aligned}
& E^{\prime}\left(U_{r}\right) C_{3} \\
& +\left(E^{\prime}\left(U_{r}\right)-E^{\prime}\left(U_{r}^{\#}\right)\right) \mathcal{F}_{r}\left(U_{l}^{\#}, U_{r}^{\#}\right)+g\left(z_{r}-z^{\#}\right) \mathcal{F}^{h}\left(U_{l}^{\#}, U_{r}^{\#}\right) \leq 0 .
\end{aligned}
$$

Now we compute

$$
\begin{aligned}
& E^{\prime}\left(U_{r}\right)-E^{\prime}\left(U_{r}^{\#}\right) \\
= & \left(g\left(h_{r}-h_{r}^{\#}\right)-\frac{a_{r}^{2}}{2}+\frac{\left(a_{r}^{\#}\right)^{2}}{2}, 0,0, a_{r}-a_{r}^{\#}, 0\right) \\
= & \left(g\left(h_{r}-h_{r}^{\#}\right)-\left(1-\kappa_{r}^{2}\right) \frac{a_{r}^{2}}{2}, 0,0,\left(1-\kappa_{r}\right) a_{r}, 0\right) .
\end{aligned}
$$

With (4.86) we obtain

$$
\begin{aligned}
& E^{\prime}\left(U_{r}\right) C_{3} \\
& +\left(E^{\prime}\left(U_{r}\right)-E^{\prime}\left(U_{r}^{\#}\right)\right) \mathcal{F}_{r}\left(U_{l}^{\#}, U_{r}^{\#}\right)+g\left(z_{r}-z^{\#}\right) \mathcal{F}^{h}\left(U_{l}^{\#}, U_{r}^{\#}\right) \\
= & g\left(h_{r}-\frac{a_{r}^{2}}{2 g}-h_{r}^{\#}+\kappa_{r}^{2} \frac{a_{r}^{2}}{2 g}+z_{r}-z^{\#}\right) \mathcal{F}^{h}\left(U_{l}^{\#}, U_{r}^{\#}\right) .
\end{aligned}
$$

According to (3.15) this will be zero if $h_{r}>0$ and $h_{r}+\left(\gamma^{2}-1\right) \frac{a_{r}^{2}}{2 g} \geq z^{\#}-z_{r}$. Otherwise we have $h_{r}^{\#}=0, \mathcal{F}^{h}\left(U_{l}^{\#}, U_{r}^{\#}\right) \geq 0$ with the term between brackets in the right-hand side of (4.91) nonpositive, which gives the inequality (4.89) and the result. The left inequality is very similar and is omitted here. 


\section{Numerical results}

In this section we perform numerical computations in order to evaluate the properties and the accuracy of the two variants of our scheme, in relation with Theorems 3.1 and 3.2. Initial data of more general type are used in [35] for the simulation of the one-dimensional shallow water MHD system with Coriolis force with the scheme presented here, extended with Coriolis force via the apparent topography method $[8$, section 4.12$]$.

First and second-order methods in time and space are evaluated here, the latter using an ENO reconstruction, as described in [8, section 4.13]. The conservative variable is $U$ as in (3.2), and the slope limitations are performed on the variables $h, h+z, u, v, h a, b$. We also compare results obtained with different values of the parameter $\gamma \geq 1$, which is a key to obtain the well-balanced property for steady states of material resonance.

The space variable $x$ is taken in $[0,1], g=9.81$, and Neumann boundary conditions are applied. We take 200 points, and plot a reference solution obtained by a second-order computation with 3300 points. The CFL-number is taken $1 / 2$ in all runs.

Test 1. Our first test includes two steady states:

- On $[0,1 / 2)$, we take initial data corresponding to a steady state in the case of material resonance of the type (2.3).

- On $(1 / 2,1]$, we take initial data corresponding to a steady state in the case of material and Alfven resonance.

Overall we have a Riemann problem at $x=0.5$. The initial data is sketched on Figure 1 and the numerical values are given in Tables 1 and 2. Figures 2 and 3 show the reference solution at time $t=0.02$ and $t=0.08$ respectively. It consists of, from left to right, a material contact, a left rarefaction wave, a left Alfven contact, a resonant material - right Alfven contact, and a right shock. The solution computed with the first scheme of Theorem 3.1 at times $t=0.02$ and $t=0.08$ is shown on Figures 4 and 5 respectively. We do not plot the results given by the second scheme of Theorem 3.2 since they are so close to the results of the first scheme that they cannot be distinguished with the eye. We observe that the second-order resolution improves the sharpness of contact discontinuities. On Figure 6 we observe that the solution computed with $\gamma=1$ looses the well-balanced property for the resonant material contact, whereas with $\gamma=2$ it is well-balanced, which is coherent with point (vii) of Theorem 3.1. Even when zooming, the results obtained with our two schemes are hardly distinguishable with the eye. Indeed for most data the two schemes from Theorems 3.1 and 3.2 give indistinguishable results. Test 2 below however shows data for which the two schemes give different results. 


\begin{tabular}{|c|cccccc|}
\hline Values of $x$ & $z$ & $h$ & $u$ & $v$ & $a$ & $b$ \\
\hline $\mathrm{x} \leq 0.2$ & 0.5 & 1.5 & 0 & 2 & $1 / \sqrt{1.5}$ & $2 / \sqrt{1.5}$ \\
\hline $0.2<\mathrm{x} \leq 0.5$ & 0 & 2 & 0 & 2 & $1 / \sqrt{2}$ & $2 / \sqrt{2}$ \\
\hline
\end{tabular}

Table 1: Initial data for Material resonance

\begin{tabular}{|c|cccccc|}
\hline Values of $x$ & $z$ & $h$ & $u$ & $v$ & $a$ & $b$ \\
\hline $0.5<x \leq 0.625$ & 0 & 0.5 & 0 & 0.5 & 0 & 1 \\
\hline $0.625<\mathrm{x} \leq 1$ & $d(x)$ & $(0.5-d(x))_{+}$ & 0 & $0.5+d(x)$ & 0 & $1+d(x)$ \\
\hline
\end{tabular}

Table 2: Initial data for Material and Alfven resonance, $d(x)=4(x-0.625)$

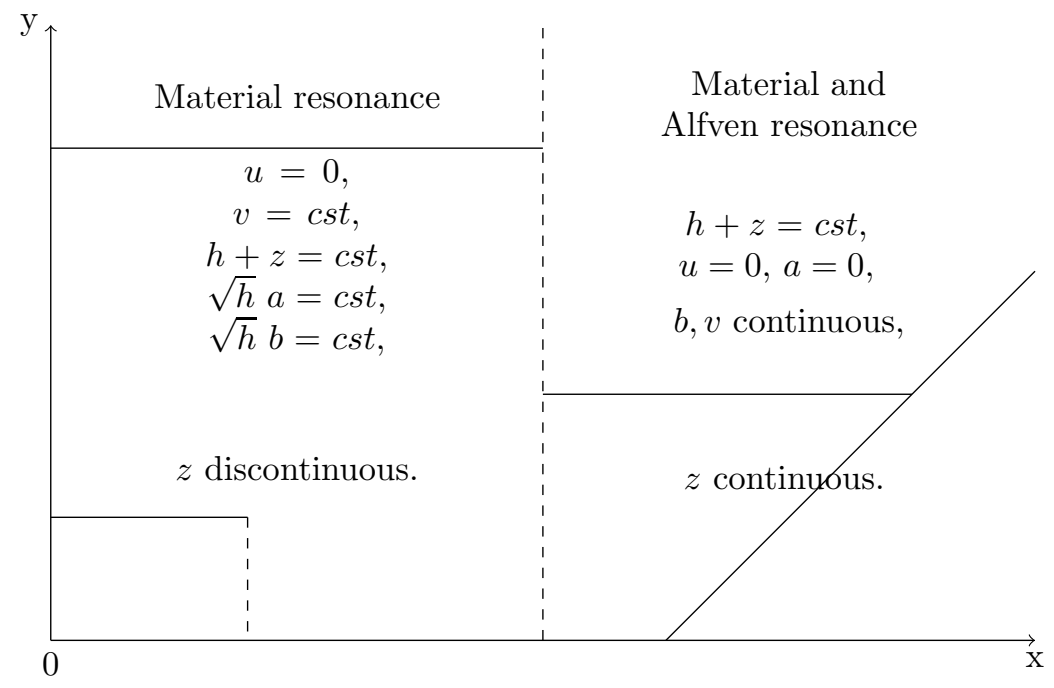

Figure 1: Initial data configuration for Test 1

Test 2. Our second test is a steady state which is a continuous material resonant contact of the type (2.4). It enables to test the long time behaviour and the stability of our two schemes. We take the values $u=0, v=2, h a=5$, $b=0$,

$$
h(x)=\frac{1}{4}+\left(\frac{1}{16}-\left(x-\frac{1}{2}\right)^{2}\right)_{+},
$$




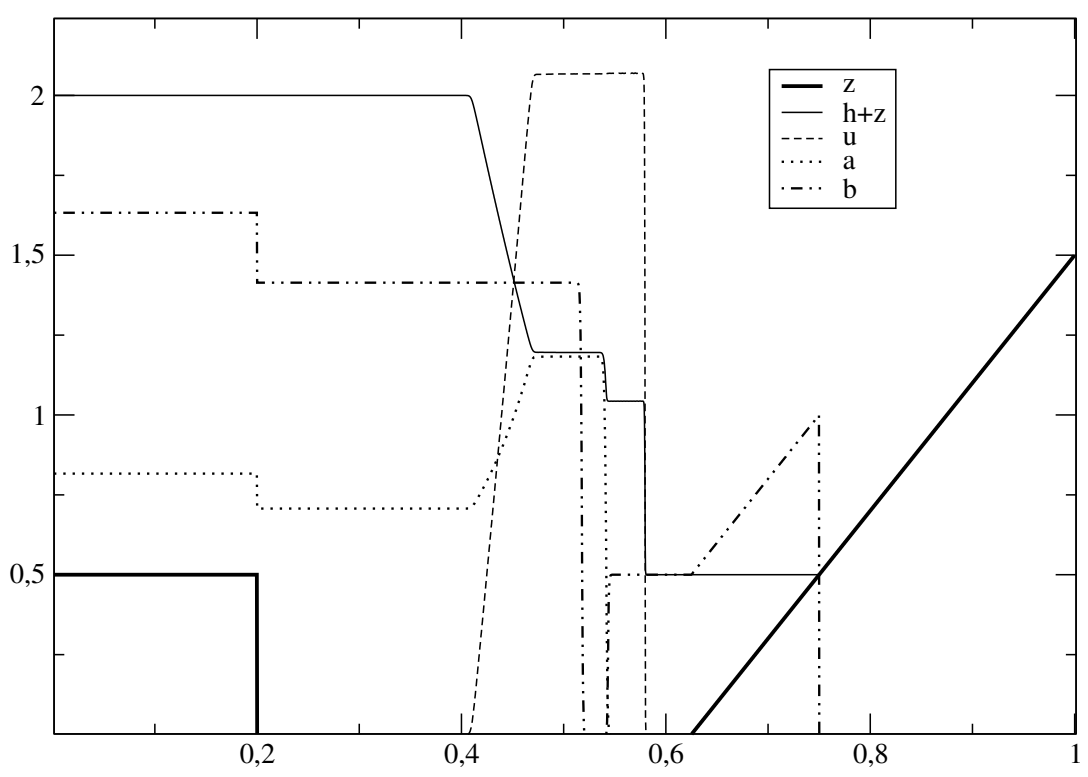

Figure 2: Reference solution for Test 1 at time $t=0.02$ computed at second order with 3300 points

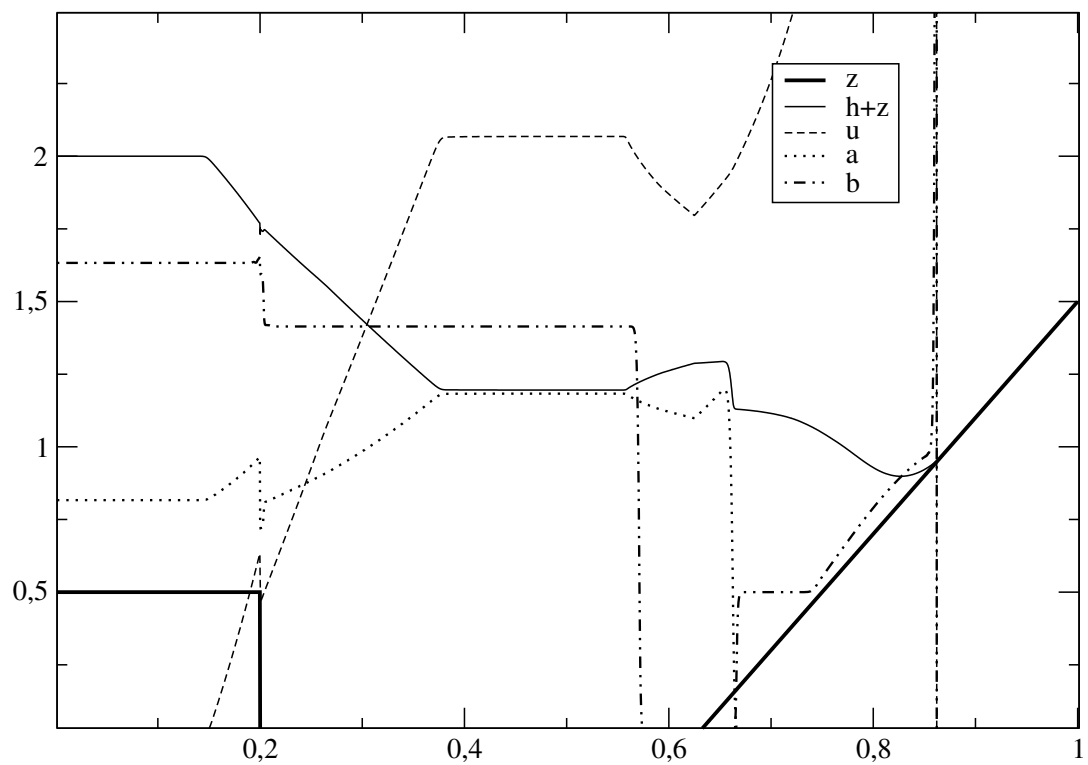

Figure 3: Reference solution for Test 1 at time $t=0.08$ computed at second order with 3300 points 

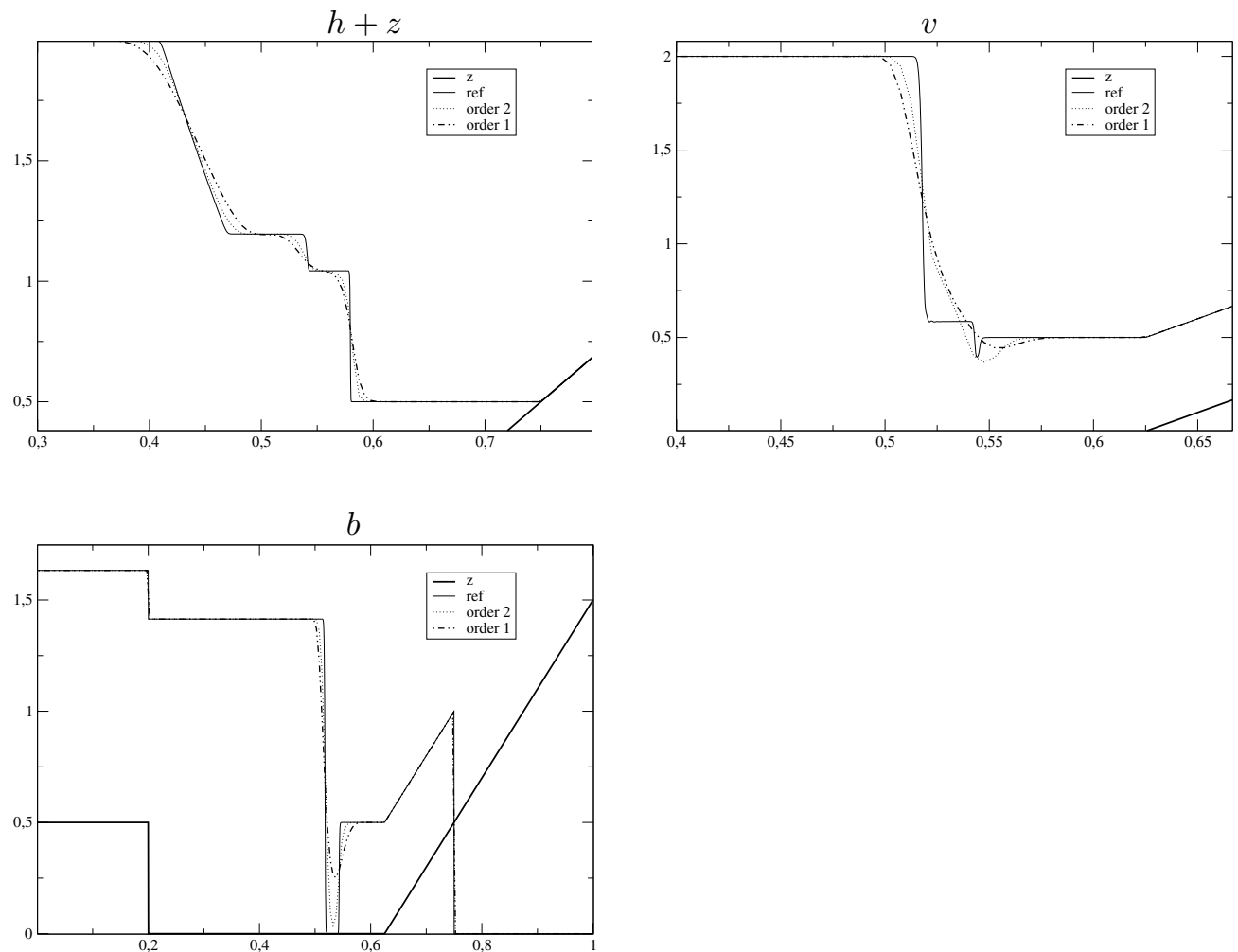

Figure 4: Components $h+z, v, b$ for Test 1 at time $t=0.02$ computed at first and second order with 200 points with the first scheme of Theorem 3.1. The reference solution is the continuous line. The second scheme of Theorem 3.2 gives almost identical results (not shown). 

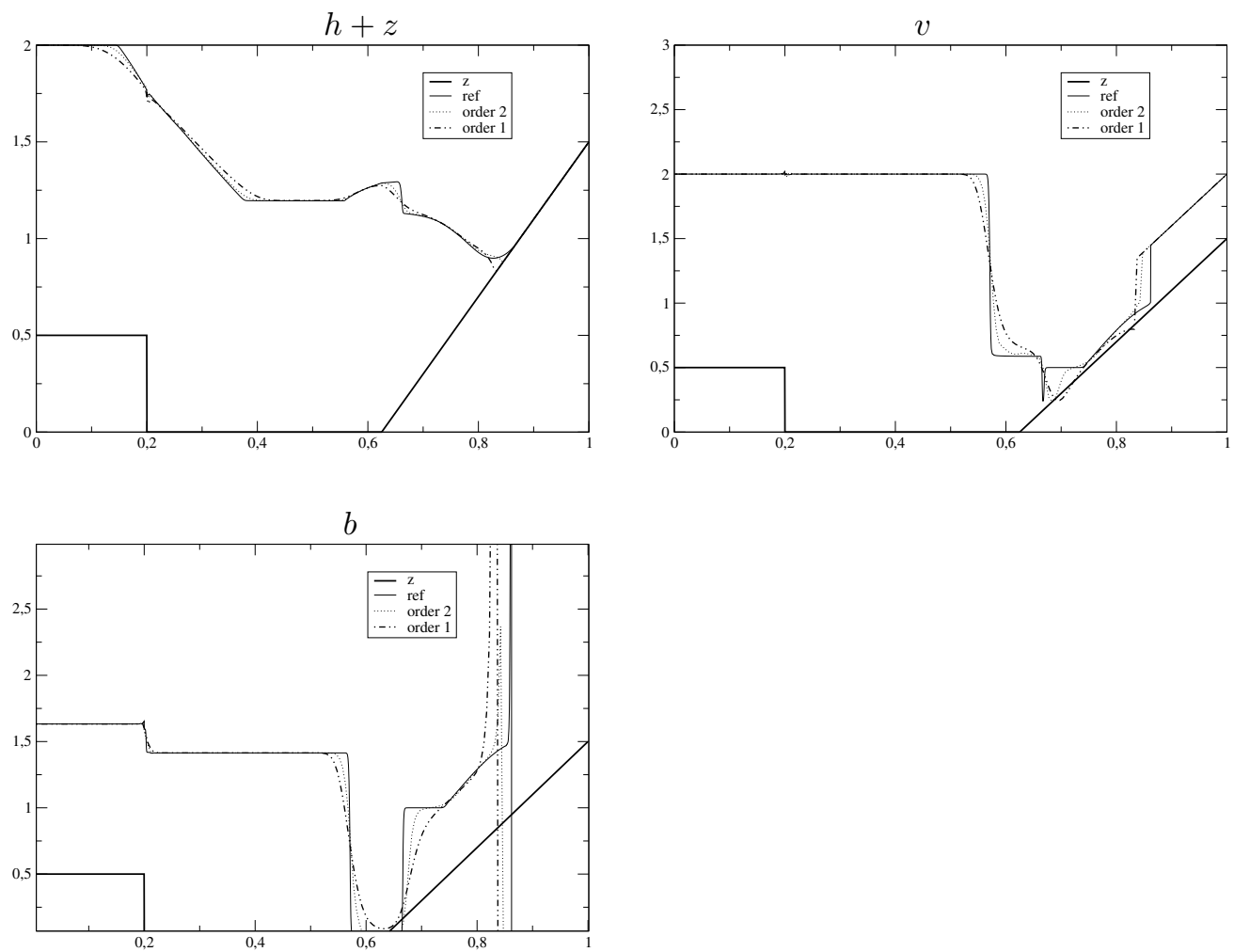

Figure 5: Components $h+z, v, b$ for Test 1 at time $t=0.08$ computed at first and second order with 200 points with the first scheme of Theorem 3.1. The reference solution is the continuous line. The second scheme of Theorem 3.2 gives almost identical results (not shown). 

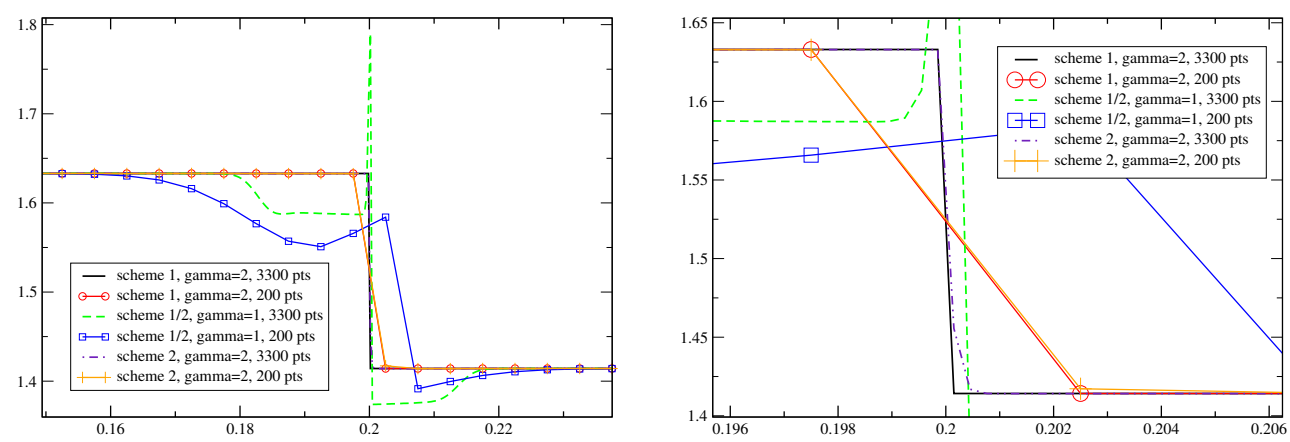

Figure 6: Zoom of component $b$ for Test 1 at the material resonance at time $t=0.02$ computed at first order, with either a high resolution of 3300 points or a low resolution of 200 points, with different values of $\gamma$, and either the first scheme of Theorem 3.1 or the second scheme of Theorem 3.2 (they are identical when $\gamma=1$ and are denoted by scheme $1 / 2)$. The value $\gamma=1$ leads to a slight overshoot while the value $\gamma=2$ does not. The right picture is a further zoom of the left one. We observe that even at the material resonance, the schemes 1 and 2 give almost the same results, the difference can only be seen on the right picture for 200 points and $\gamma=2$. The scheme 1 gives the exact solution, in accordance with Theorem 3.1(vii) since here the contact discontinuity is of the type (2.3). The scheme 2 does not give the exact solution but is nevertheless extremely accurate.

and $z(x)$ is defined to satisfy $h-a^{2} / 2 g+z=c s t$ while taking the value 0 at the boundary, that is

$$
z(x)=\frac{25}{2 g h(x)^{2}}-h(x)+c, \quad c=\frac{1}{4}-\frac{16 \times 25}{2 g} .
$$

We run our two schemes 1 and 2 respectively from Theorems 3.1 and 3.2, at first-order with 200 points in space, until the time $t=1$, and with the choice $\gamma=2$. We also run a splitting scheme that solves on one hand the system (1.5)-(1.9) without topography with the solver of [10], and on the other hand the topography alone by the simple centered difference scheme $u_{i}^{n+1}=u_{i}^{n}-$ $\Delta \operatorname{tg}\left(z_{i+1}-z_{i-1}\right) / 2 \Delta x$. We call this scheme 0 .

Figure 7 shows the values of $h+z, u$, ha for the schemes 1,2, 0. The computation takes 14458 timesteps for scheme 1, 8025 for scheme 2, and 8435 for scheme 0 . We observe that scheme 2 gives the exact solution, which is the initial data. Scheme 1 gives a wrong solution that concentrates all the mass in the middle of the box. It is a kind of steady solution different from the initial data, and we notice that the value of $h a$ has not been preserved. The scheme 0 gives a quite good solution except that the velocity $u$ is wrong, it takes a value of approximately 2 instead of 0 . We have observed (not shown here) that this value is indeed correlated to the given value of $v$.

In order to understand the asymptotic behaviour we plot on Figure 8 the time evolution of the $L^{1}$ norm of $h u$, for schemes 1 and 0 . Since $a \sim 20$, during the whole simulation the waves can travel 20 times the length of the box. For 
scheme 1 we observe that a (wrong) steady solution is reached after some time. When refining the grid the solution takes more and more time to leave the initial steady state and to reach the wrong steady state. Convergence to the exact solution is thus recovered when the grid size tends to 0 . For a grid of 1600 points the exact solution is indeed kept until the time $t=1$ (not shown). For scheme 0 (splitting scheme) we observe a linear growth of the velocity with time, that makes the computed solution definitely leave the initial steady state. The situation is not improved when refining the grid.
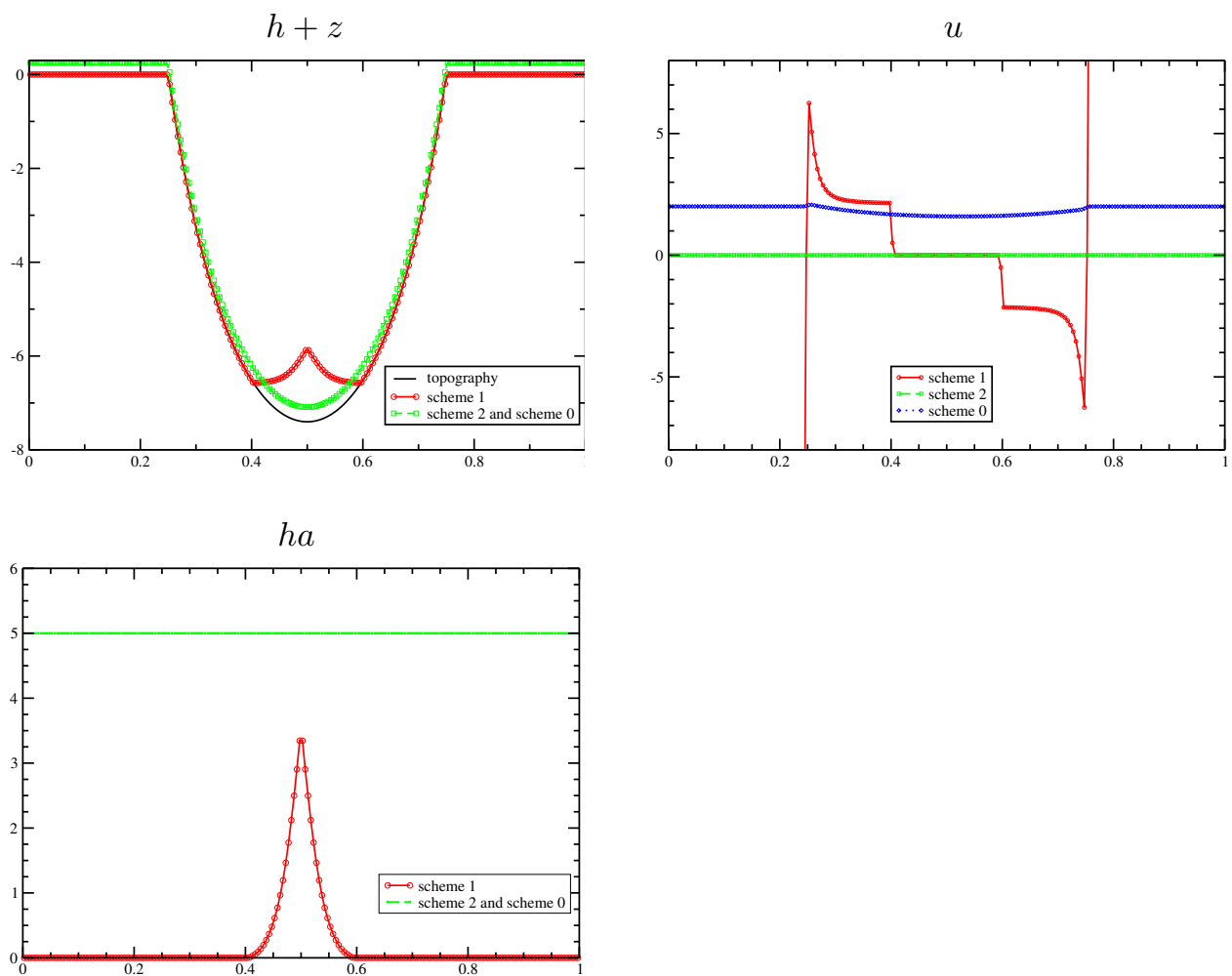

Figure 7: Components $h+z, u$, ha for Test 2 with schemes 1, 2, 0. with 200 points in space, at time $t=1$. Scheme 2 gives the exact solution, identical to the initial data. Scheme 1 gives a wrong solution, a kind of steady solution different from the initial data (the value of $h a$ has not been preserved), and scheme 0 gives a quite good solution except that the velocity $u$ is wrong, it takes the value of approximately 2 instead of 0 . Note that $h$ vanishes outside the center of the box for scheme 1 , thus the values of $u$ and $a$ outside this center region are irrelevant. 

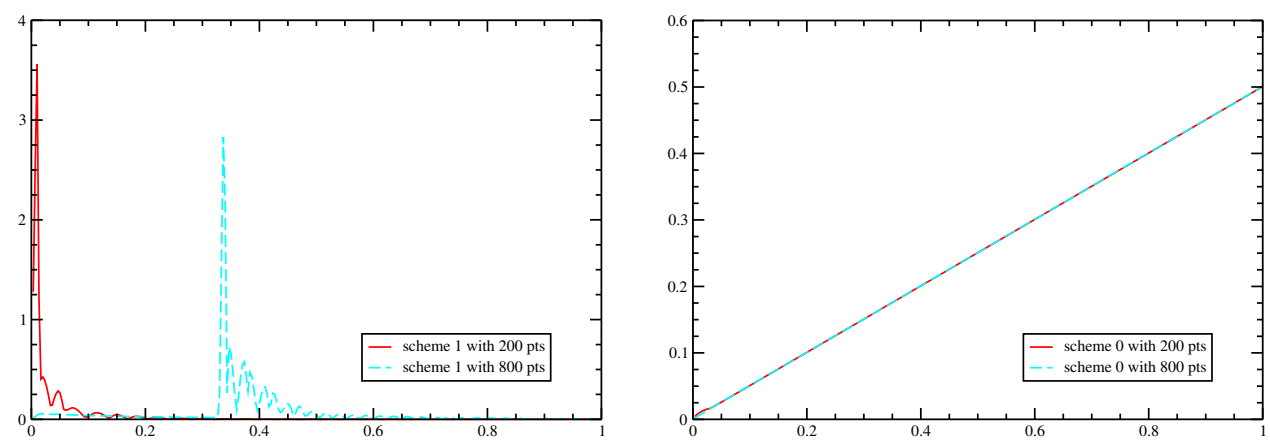

Figure 8: Time evolution of the $L^{1}$ norm of $h u$ for Test 2. Left: scheme 1 for respectively 200 and 800 points in space. The peak shows that the computed solution leaves the initial steady state. It then converges to the wrong steady state, that nevertheless satisfies $h u=0$. The peak appears later when the grid is refined, so that the exact initial steady state is recovered in the limit of a vanishing grid size, while keeping a fixed time interval. Right: scheme 0 for respectively 200 and 800 points in space. The computed solution never reaches a steady state, and the situation does not improve when refining the grid.

\section{Conclusion}

We have proposed two variants of a hydrostatic reconstruction scheme for the one-dimensional shallow water magnetohydrodynamic system with topography. They are multi well-balanced in the sense that they exactly solve several families of steady states. The two schemes differ by the material resonant steady state that they resolve exactly. Scheme 1 exactly solves the steady states (2.3), while scheme 2 exactly solves the steady states (2.4) (see Theorems 3.1 and 3.2). Both exactly solve the steady states (2.6), and also the steady material and Alfven contact discontinuities when there is no jump in topography. They keep $h$ nonnegative, and satisfy a semi-discrete entropy inequality. They involve a cutoff parameter $\gamma$ that enables to keep the propagation speed finite. Scheme 2 has overall the property to preserve the relation $h a=c s t$ if it is satisfied initially

Well-balanced schemes are known to perform better than classical schemes (like the splitting of the topography for example), as many studies have proved for the shallow water system. Our schemes take into account this knowledge since they generalize the hydrostatic reconstruction scheme of [1], that is recovered when the magnetic field vanishes.

Numerical tests show that both schemes give extremely close results on most data. In particular they give very accurate results on contact waves. Steady states of the form (2.3) are exactly resolved by scheme 1, and are resolved with excellent accuracy by scheme 2 . Steady states of the form (2.4) are exactly resolved by scheme 2, but give however slightly unstable results when using scheme 1. Scheme 1 nevertheless recovers the exact steady state in this case when refining the grid, which is not the case when using a splitting method. Our overall evaluation is thus that scheme 2 gives slightly better results than 
scheme 1.

\section{References}

[1] E. Audusse, F. Bouchut, M.-O. Bristeau, R. Klein, B. Perthame, A fast and stable well-balanced scheme with hydrostatic reconstruction for shallow water flows, SIAM J. Sci. Comput. 25 (2004), 2050-2065.

[2] E. Audusse, F. Bouchut, M.-O. Bristeau, J. Sainte-Marie, Kinetic entropy inequality and hydrostatic reconstruction scheme for the Saint-Venant system, Math. Comput. 85 (2016), 2815-2837.

[3] D.S. Bale, R.J. Leveque, S. Mitran, J.A. Rossmanith, A wave propagation method for conservation laws and balance laws with spatially varying flux functions, SIAM J. Sci. Comput. 24 (2002), 955-978.

[4] D.S. Balsara, M. Dumbser, R. Abgrall, Multidimensional HLL and HLLC Riemann solvers for unstructured meshes with application to Euler and MHD flows, J. Comput. Phys. 261 (2014), 172-208.

[5] D.S. Balsara, D.S. Spicer, A staggered mesh algorithm using high order Godunov fluxes to ensure solenoidal magnetic fields in magnetohydrodynamic simulations, J. Comput. Phys. 149 (1999), 270-292.

[6] C. Berthon, C. Chalons, A fully well-balanced, positive and entropysatisfying Godunov-type method for the shallow-water equations, Math. Comput. 85 (2016), 1281-1307.

[7] C. Berthon, F. Foucher, Efficient well-balanced hydrostatic upwind schemes for shallow water equations, J. Comput. Phys. 231 (2012), 4993-5015.

[8] F. Bouchut, Nonlinear stability of finite volume methods for hyperbolic conservation laws, and well-balanced schemes for sources, Birkhäuser, 2004.

[9] F. Bouchut, S. Boyaval, A new model for shallow viscoelastic fluids, Math. Models and Meth. in Appl. Sci. 23 (2013), 1479-1526.

[10] F. Bouchut, X. Lhébrard, A 5-wave relaxation solver for the shallow water MHD system, J. Scientific Comput. 68 (2016), 92-115.

[11] F. Bouchut, T. Morales, A subsonic-well-balanced reconstruction scheme for shallow water flows, Siam J. Numer. Anal. 48 (2010),1733-1758.

[12] F. Bouchut, V. Zeitlin, A robust well-balanced scheme for multi-layer shallow water equations, Discrete and Continuous Dynamical Systems B, 13 (2010), 739-758.

[13] M.J. Castro, E.D. Fernández-Nieto, A class of computationally fast first order finite volume solvers: PVM methods, SIAM J. Sci. Comput. 34 (2012), 2173-2196.

[14] M.J. Castro, A. Pardo, C. Parés, Well-balanced numerical schemes based on a generalized hydrostatic reconstruction technique, Math. Models Methods Appl. Sci. 17 (2007), 2065-2113. 
[15] M.J. Castro, A. Pardo, C. Parés, E.F. Toro, On some fast well-balanced first order solvers for nonconservative systems, Math. Comput. 79 (2010), 1427-1472.

[16] C. Chalons, F. Coquel, E. Godlewski, P.-A. Raviart, N. Seguin, Godunovtype schemes for hyperbolic systems with parameter-dependent source. The case of Euler system with friction, Math. Models Methods Appl. Sci. 20 (2010), 2109-2166.

[17] A. Chertock, A. Kurganov, Y. Liu, Central-upwind schemes for the system of shallow water equations with horizontal temperature gradients, Num. Math. 127 (2014), 595-639.

[18] A. Dedner, F. Kemm, D. Kröner, C.-D. Munz, T. Schnitzer, M. Wesenberg, Hyperbolic divergence cleaning for the MHD equations, J. Comput. Phys. 175 (2002), 645-673.

[19] P.J. Dellar, Hamiltonian and symmetric hyperbolic structures of shallow water magnetohydrodynamics, Physics of Plasmas 9 (2002), 1130.

[20] H. DeSterck, Hyperbolic theory of the shallow water magnetohydrodynamics equations, Physics of Plasmas 8 (2001), 3293.

[21] V. Desveaux, M. Zenk, C. Berthon, C. Klingenberg, Well-balanced schemes to capture non-explicit steady states: Ripa model, Math. Comput. 85 (2016), 1571-1602.

[22] V. Desveaux, M. Zenk, C. Berthon, C. Klingenberg, A well-balanced scheme to capture non-explicit steady states in the Euler equations with gravity, Intern. J. Numer. Meth. Fluids 81 (2016), 104-127.

[23] F.G. Fuchs, A.D. McMurry, S. Mishra, N.H. Risebro, K. Waagan, Approximate Riemann solvers and robust high-order finite volume schemes for multi-dimensional ideal MHD equations, Comm. Comput. Phys. 9 (2011), 324-362.

[24] D.L. George, Augmented Riemann solvers for the shallow water equations over variable topography with steady states and inundation, J. Comput. Phys. 227 (2008), 3089-3113.

[25] P.A. Gilman, Magnetohydrodynamic "shallow water" equations for the solar tachocline, Astrophys. J. Lett. 544 (2000), L79-L82.

[26] C. Parés, Numerical methods for nonconservative hyperbolic systems: a theoretical framework, SIAM J. Numer. Anal. 44 (2006), 300-321.

[27] C. Parés, M. Castro, On the well-balance property of Roe's method for nonconservative hyperbolic systems. Applications to shallow-water systems, Math. Modelling Num. Anal. 38 (2004), 821-852.

[28] S. Qamar, G. Warnecke, Application of space-time CE/SE method to shallow water magnetohydrodynamic equations, J. Comput. Appl. Math. 196 (2006), 132-149. 
[29] J.A. Rossmanith, A constrained transport method for the shallow water MHD equations, in Hyperbolic problems: theory, numerics, applications, 9th International Conference on Hyperbolic Problems, Pasadena, 2002, Ed. T.Y. Hou, E. Tadmor, 851-860, 2003.

[30] J.A. Rossmanith, D.S. Bale, R.J. Leveque, A wave propagation algorithm for hyperbolic systems on curved manifolds, J. Comput. Phys. 199 (2004), 631-662.

[31] K. Waagan, C. Federrath, C. Klingenberg, A robust numerical scheme for highly compressible magnetohydrodynamics: Nonlinear stability, implementation and tests, J. Comput. Phys. 230 (2011), 3331-3351.

[32] Y. Xing, C.-W. Shu, A survey of high order schemes for the shallow water equations, J. Math. Study 47 (2014), 221-249.

[33] Y. Xing, C.-W. Shu, S. Noelle, On the advantage of well-balanced schemes for moving-water equilibria of the shallow water equations, J. Sci. Comput. 48 (2011), 339-349.

[34] V. Zeitlin, Remarks on rotating shallow-water magnetohydrodynamics, Nonlinear Processes in Geophysics 20 (2013), 893-898.

[35] V. Zeitlin, C. Lusso, F. Bouchut, Geostrophic vs magneto-geostrophic adjustment and nonlinear magneto-inertia-gravity waves in rotating shallow water magnetohydrodynamics, Geophys. Astrophys. Fluid Dynamics 109 (2015), 497-523. 